\title{
Room-Temperature Amplification of Terahertz Radiation by Grating-Gate Graphene Structures
}

\author{
Stephane Boubanga-Tombet $\odot,{ }_{1}^{1}$ Wojciech Knap $\odot,{ }^{1,2,3}$ Deepika Yadav $\odot,{ }^{1}$ Akira Satou $\odot,{ }^{1}$ Dmytro B. But $\odot,{ }^{2,4}$ \\ Vyacheslav V. Popov ${ }^{5}$ Ilya V. Gorbenko® ${ }^{6}$ Valentin Kachorovskii®, ${ }^{2,6}$ and Taiichi Otsuji® ${ }^{1, *}$ \\ ${ }^{1}$ Research Institute of Electrical Communication, Tohoku University, Sendai 980-8577, Japan \\ ${ }^{2}$ CENTERA Laboratories, Institute of High Pressure Physics PAS, Warsaw 01-142, Poland \\ ${ }^{3}$ Laboratory Charles Coulomb, University of Montpellier and CNRS, Montpellier F-34095, France \\ ${ }^{4}$ CEZAMAT Warsaw Technical University, Warsaw 02-346, Poland \\ ${ }^{5}$ Kotelnikov Institute of Radio Engineering and Electronics (Saratov Branch), \\ RAS, Saratov 410019, Russia \\ ${ }^{6}$ Ioffe Institute, 194021 St. Petersburg, Russia
}

(Received 13 February 2020; revised 7 May 2020; accepted 20 May 2020; published 6 July 2020)

\begin{abstract}
We study terahertz $(\mathrm{THz})$ radiation transmission through grating-gate graphene-based nanostructures. We report on room-temperature $\mathrm{THz}$ radiation amplification stimulated by current-driven plasmon excitation. Specifically, with an increase of the dc current under periodic charge density modulation, we observe a strong redshift of the resonant $\mathrm{THz}$ plasmon absorption, followed by a window of complete transparency to incoming radiation and subsequent amplification and blueshift of the resonant plasmon frequency. Our results are, to the best of our knowledge, the first experimental observation of energy transfer from dc current to plasmons leading to $\mathrm{THz}$ amplification. Additionally, we present a simple model offering a phenomenological description of the observed $\mathrm{THz}$ amplification. This model shows that in the presence of a dc current the radiation-induced correction to dissipation is sensitive to the phase shift between oscillations of carrier density and drift velocity. And, with an increasing current, the dissipation becomes negative, leading to amplification. The experimental results of this work, as all obtained at roomtemperature, pave the way toward the new 2D plasmon-based, voltage-tunable $\mathrm{THz}$ radiation amplifiers.
\end{abstract}

\section{INTRODUCTION}

More than 40 years ago, active theoretical and experimental studies of plasma oscillations in two-dimensional electron systems began, and plasmonic resonances were observed [1-7]. The interest in this area dramatically increased after the seminal work of Dyakonov and Shur [8], who theoretically predicted that the dc current in the channel of a submicrometer-size field-effect transistors (FETs) could become unstable, leading to the excitation of plasma oscillations, with the frequency controlled by the gate voltage, and generation of tunable terahertz $(\mathrm{THz})$ radiation.

This work, as well as the next publication [9] which was focused on the plasmon-mediated $\mathrm{THz}$ detection, aroused great interest because of their novelty in the field of the

\footnotetext{
*otsuji@riec.tohoku.ac.jp
}

Published by the American Physical Society under the terms of the Creative Commons Attribution 4.0 International license. Further distribution of this work must maintain attribution to the author(s) and the published article's title, journal citation, and DOI. fundamental physics and important potential applications in $\mathrm{THz}$ optoelectronics for the creation of all-electronic, compact, and gate-tunable $\mathrm{THz}$ detectors and emitters. However, numerous experimental attempts to realize efficient, narrow-band, and voltage-tunable 2D plasmon-based detectors or emitters of $\mathrm{THz}$ radiation with single FETs have failed, as the intensity of radiation turned out to be too small and plasma resonances were too broad and/or not gate-voltage tunable [10-18].

It did not take long to understand that multiple-gate periodic structures are more promising. Such structures interact much better with $\mathrm{THz}$ radiation than the singlegate structures. In fact, the study of the grating-gatecoupler-based plasmon excitation in two-dimensional electron gases by incident $\mathrm{THz}$ waves has begun a long time ago since the seminal works of Refs. [2,3]. By using such structures, high-quality factor plasmon resonances in absorption could be excited, as demonstrated much later by Muravjov et al. [19], for 2D grating-gate GaN/AlGaN structures. The grating-gate structures also show excellent characteristics as $\mathrm{THz}$ radiation broadband detectors [20-22]. Nevertheless, despite tremendous efforts, neither the room-temperature resonant detection nor the currentstimulated emission or amplification of $\mathrm{THz}$ radiation 
have been observed so far (see the discussion in the recent work of Ref. [23], where low-temperature $\mathrm{THz}$ emission is discussed).

Fundamentally new aspects have been brought by the use of graphene-based structures [24,25]. Graphene shows record mobility at room-temperature, which gives a much higher quality factor of plasma resonances than that in conventional materials. Moreover, the mediation of plasmons can greatly enhance the interaction between light and a graphene substance due to a relatively low level of losses and a high degree of spatial electric field confinement. This result explains the growing interest in graphene plasmonics [26-33]. Plasmon-enhanced $\mathrm{THz}$ graphene devices have recently been investigated [34,35], and improvement of the device performances on gain modulation [35,36], sensitivity [37], and emission [34] have already been demonstrated.

Recently, some theoretical and experimental studies of grating-gate graphene structures utilizing plasma resonances have been reported [38-40]. In particular, the electromagnetic simulations of the graphene gratinggate structures [41] show the possibility of the resonant plasmons strongly coupled to $\mathrm{THz}$ radiation even at roomtemperature. These works show that (i) the plasmon resonances can be excited in graphene-based periodic structures in a reflectivity configuration and (ii) such a configuration can be used to obtain the $\mathrm{THz}$ radiation absorption by plasmons.

Despite intensive studies of the $\mathrm{THz}$ resonant detection in graphene-based periodic structures, there has not yet been any detailed study of current-induced effects. Also, there does not exist any report of room-temperature experiments showing efficient energy transfer from dc current to plasmons that could lead to $\mathrm{THz}$ radiation generation or amplification [42-44].

In this work, we explore $\mathrm{THz}$ light-plasmon coupling, light absorption, and amplification by graphene gratinggate structures focusing on current-driven effects. These grating-gate structures are used to create a periodic structure of highly conducting active regions separated by lowconducting passive regions. The large difference between concentrations allows us to localize plasmons in active regions and control their properties both by gate electrodes and by the dc driving current. We demonstrate that, in such structures, gate-voltage-controlled resonant plasmons are excited by $\mathrm{THz}$ radiation and show current-driven amplification of this radiation. More specifically, we show that with the increase of dc current the plasmon spectra undergoes a strong redshift, followed by a complete lack of resonant $\mathrm{THz}$ plasmon absorption and subsequent amplification with a clear blueshift.

We also present a phenomenological theoretical description of observed results using a simple model of periodically alternated stripes of the high (low) electron density and corresponding high (low) plasma wave velocity. We show that, in such a plasmonic crystal structure, the $\mathrm{THz}$ radiation dissipation becomes sensitive to the phase shift between the oscillations of carrier density and drift velocity and that, with increasing the dc current, the radiationrelated correction to dissipation changes its sign, resulting in amplification of the optical signal.

Our main results can be formulated as follows: (i) experiments demonstrating that the current-driven plasmon resonances at the $\mathrm{THz}$ range, with the increase of the current, undergo a redshift, followed by a window of complete transparency to incoming radiation and subsequent amplification and blueshift of the resonant plasmon frequency, and (ii) theory providing the phenomenological description of the experimentally observed phenomena, in particular, switching from dissipation to amplification at drift velocities smaller than the plasma wave velocity.

Importantly, all experimental results are obtained at roomtemperature and, therefore, can be used for the design of graphene- or semiconductor-based resonant, compact, and voltage- or current-controlled $\mathrm{THz}$ absorbers and amplifiers as well as coherent sources.

\section{EXPERIMENTAL}

\section{A. Samples}

The samples are fabricated with a field-effect transistor structure featuring an interdigitated dual-grating-gate (DGG) where the plasmonic cavities are formed below the gate electrode grating fingers [21,45-47]. In order to ensure high carrier mobility in our devices, $h$-BN-encapsulated graphene heterostructures ( $h$-BN/graphene/ $h$-BN) are fabricated. The optically estimated $h$-BN thickness is in the range from 20 to $32 \mathrm{~nm}$ for both structures. The first $h$-BN layer is transferred onto a $\mathrm{SiO}_{2} / \mathrm{Si}$ wafer serving as a part of the back-gate dielectric layer. The process is followed by transferring the monolayer graphene. The second $h$-BN layer is transferred onto the monolayer graphene and serves as the top-gate dielectric layer. The layer sequence and the sample architecture are shown in Fig. 1. Raman spectroscopy (sharp mono peak at the $G$ and $G^{\prime}$ bands) and the contrast fractions of the optical microscope images of the transferred graphene sheets allow confirming that they are monolayers.

Two device structures are designed with a gate finger width of $L_{g 1}=0.5 \mu \mathrm{m}$ and $L_{g 2}=1 \mu \mathrm{m}\left(L_{g 1}=0.75 \mu \mathrm{m}\right.$ and $L_{g 2}=1.5 \mu \mathrm{m}$ ) separated by $d_{1}=0.5 \mu \mathrm{m}$ and $d_{2}=$ $1 \mu \mathrm{m}$ gaps $\left(d_{1}=0.5 \mu \mathrm{m}\right.$ and $\left.d_{2}=2 \mu \mathrm{m}\right)$ referred to as asymmetric DGG structures (A-DGG 3.1 and A-DGG 3.2, respectively) (see Fig. 1). The devices have very similar electrical properties with very close charge neutrality points (CNPs), ranging from -0.1 to $+0.15 \mathrm{~V}$.

In the experiments, we manipulate the electron concentration in the channel by gate voltages to create active and passive plasmonic regions with high and low electron concentration, respectively. Specifically, we always bias 


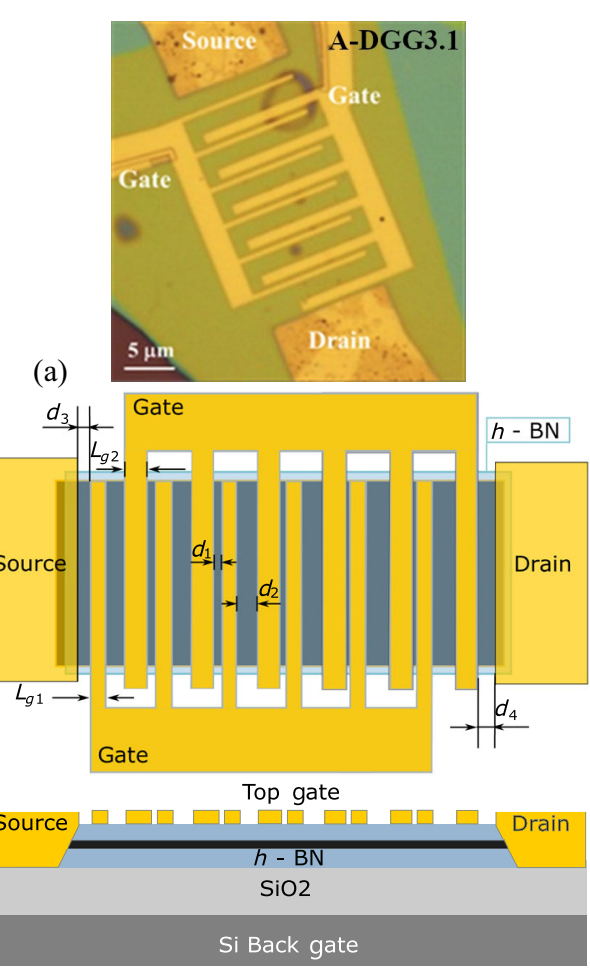

(b)

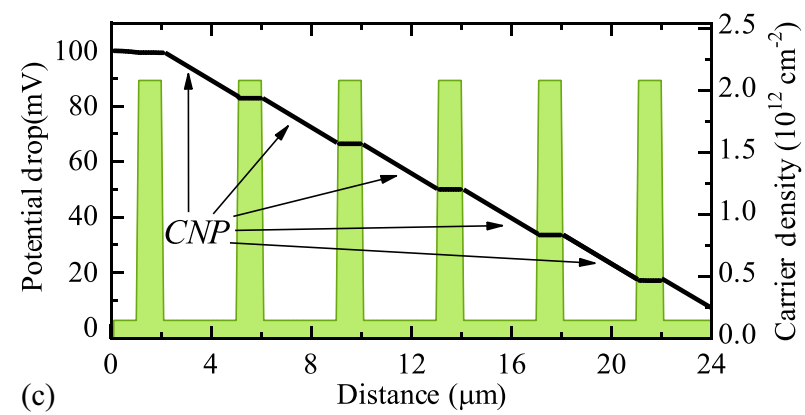

FIG. 1. The microphoto of the A-DGG3.1 sample (a), schematic top and cross-sectional device images illustrating the $h$-BN/ graphene $/ h$-BN heterostructure including the asymmetric dual grating-gate metallization (b), and the gate-controlled charge density distribution (green area) together with potential distribution along the channel (black solid line) (c). the gates in such a way that, in a given experiment configuration, only one type of gate is positively biased (electrical $n$-type doping) and all other types of gates are set at voltages granting the CNP condition of the graphene layer.

In this way, by successive biasing the different gates, we define four types of grating-gate structure $(C 1, \ldots, C 4)$ with cavities of length $0.5,0.75,1.0$, and $1.1 \mu \mathrm{m}$, each composed of six fingers with highly doped $n$-type cavities (up to approximately $2 \times 10^{12} \mathrm{~cm}^{-2}$ ) separated by six regions at the CNP condition $\left(n_{2} \sim 0.1 \times 10^{12} \mathrm{~cm}^{-2}\right.$ for both electrons and holes at room-temperature). The parameters of these structures are given in Table I. The example of typical carrier density distribution in the experiments on cavity $C 2$ is shown in Fig. 1(c). We also show an example of the potential distribution in the case of application of $100 \mathrm{mV}$ drain-to-source voltage [Fig. 1(c)].

The example of the results of electrical characterization (sample A-DGG 3.1) is shown in Fig. 2. The multiple sweeps of gate voltages show only minimal effects of hysteresis (see Fig. 2) and grant good reproducibility of all the experimental results.

In Figs. 2(a) and 2(b), we also present the sample resistance as a function of the inverse swing voltage, $1 /\left(U_{g}-U_{\mathrm{CNP}}\right)$, allowing one to determine the parasitic resistance $R_{0}$, i.e., the sum of the contact resistances and resistances of ungated CNP biased parts of each sample. One can see that, as expected from the sample architecture, the biasing of the front gates changes the resistance in a much smaller degree than the back gating. The resistance change is roughly proportional to the ratio of the length of the biased gate $(\times 6)$ and the total length of the channel, as given in Table I. Knowing the total parasitic resistance $R_{0}$, we can extract sample averaged conductivity $\sigma=\left(L_{\mathrm{ch}} / W\right) /$ $\left(R-R_{0}\right)$, where $L_{\mathrm{ch}}$ is the total channel length, $W$ is the average channel width, and $R$ is the total resistance. The carrier mobility $\mu_{i}$ can be calculated from the equation $e \mu_{i}\left(n_{i}+p_{i}\right)=\sigma_{i}$ (see Refs. [29,48] for details). The electron and hole density $n_{i}$ and $p_{i}$ (index $i=1,2$ numerates regions with high and low conductivity,

TABLE I. Samples and different plasmonic cavity parameters.

\begin{tabular}{lcccc}
\hline \hline Cavity & $C 1$ & $C 2$ & $C 3$ & $C 4$ \\
\hline Structure or sample & A-DGG3.1 & A-DGG3.2 & A-DGG3.1 & A-DGG3.2 \\
Thickness of top $h$-BN layer $(\mathrm{nm})$ & 32 & 20 & 32 & 20 \\
CNP $(\mathrm{V})$ & +0.15 & -0.12 & +0.10 & -0.06 \\
Biased cavity length $(\mu \mathrm{m})$ & 0.5 & 0.75 & 1.0 & 1.5 \\
Total channel length $(\mu \mathrm{m})$ & 26.5 & 24.0 & 26.5 & 24.0 \\
$d_{1}$ and $d_{2}(\mu \mathrm{m})$ & 0.5 and 2.0 & 0.5 and 1.0 & 0.5 and 2.0 & 0.5 and 1.0 \\
Channel width (average) $(\mu \mathrm{m})$ & 4.9 & 1.325 & 4.9 & 1.325 \\
$d_{3}$ and $d_{4}(\mu \mathrm{m})$ & 2.0 and 0.5 & 1.0 and 0.5 & 2.0 and 0.5 & 1.0 and 0.5 \\
\hline \hline
\end{tabular}



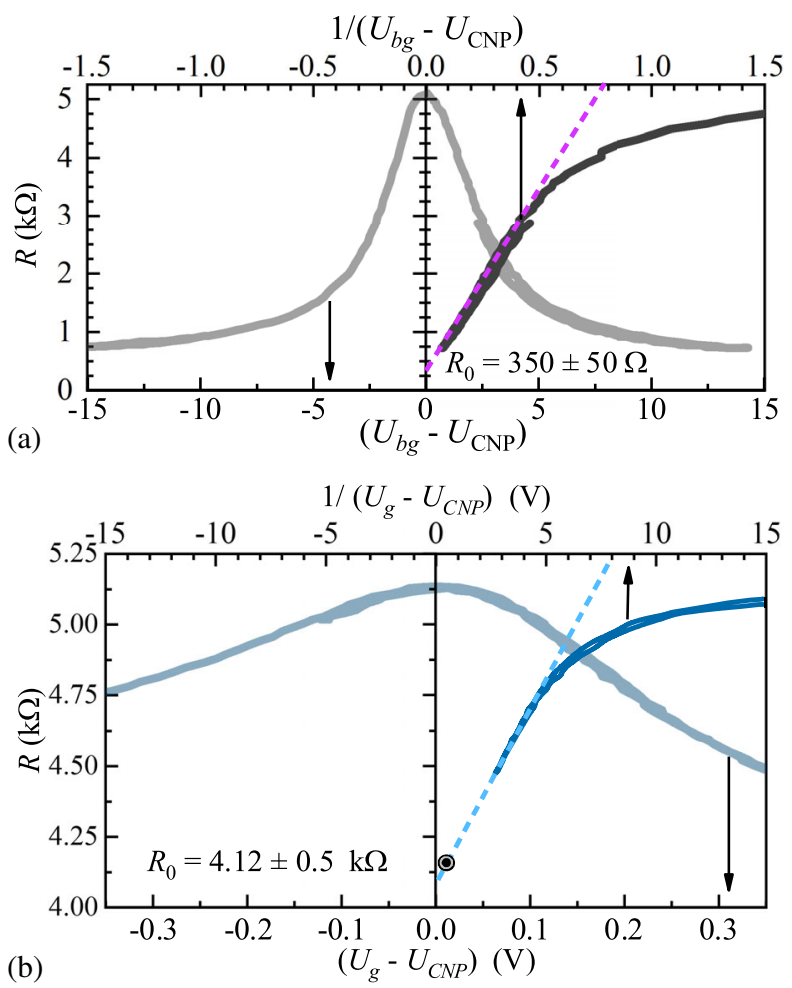

(b)

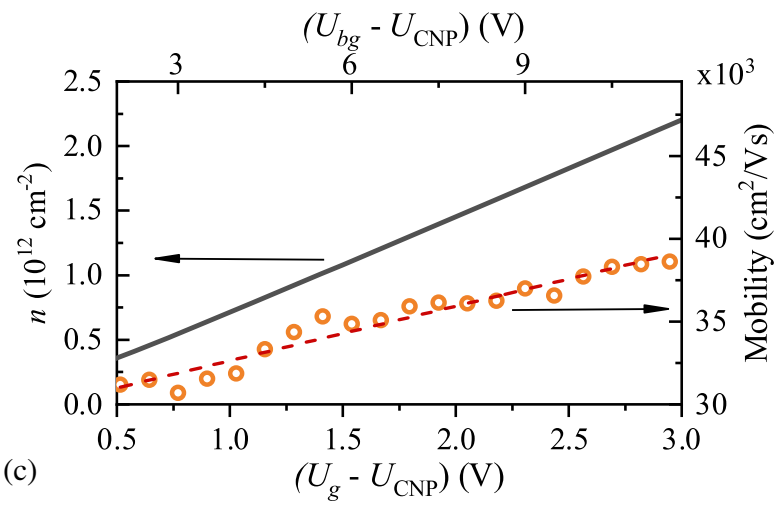

FIG. 2. Electrical characterization of the samples. Ambipolar resistance versus back gate (a) and top gate for the $C 1$ cavity (b) of the A-DGG3.1 structure. We also show resistance versus inverse swing voltage $\left[1 /\left(U_{g}-U_{\mathrm{CNP}}\right]\right.$ that allows extraction of the resistance of the contacts (a) and the resistance of total ungated part of samples for the $C 1$ cavity (b). $C 1$ cavity carrier density $n$ and mobility as a function of the gate voltage (c): The lower horizontal axis corresponds to the top-gate bias, and the upper horizontal axis is for the back-gate bias.

respectively) as a function of the back-gate voltage can be calculated using the parallel-plate capacitor model with the correction by the quantum capacitance of graphene. Figure 2(c) shows the carrier density and mobility as a function of the front-gate voltage (lower horizontal scale) and back-gate voltage (upper horizontal scale). As can be seen, the mobility is over $30000 \mathrm{~cm}^{2} / \mathrm{V} \mathrm{s}$ in the whole investigated range, indicating the excellent quality of $h$-BN-encapsulated graphene.

\section{B. Time-domain spectroscopy experiments}

We use in our experiment grating-gate-based structures. Such structures have been used to study the plasmon excitation in two-dimensional electron gas by an incident $\mathrm{THz}$ wave since the pioneering works of Allen and Theis $[2,3]$. In particular, recently, the grating-gate structures were used in graphene plasmons experimental studies [40]. Also, the electromagnetic simulation of plasmon excitation by a $\mathrm{THz}$ wave in graphene with an asymmetric grating gate (which is very similar to the structure measured in our paper) was recently presented in Ref. [41]. These works show that the plasmon resonances can be excited in gratinggate graphene structures in a reflectivity configuration and can be used to obtain the $\mathrm{THz}$ radiation absorption.

The experimental setup configuration is shown in Fig. 3(a). THz time-domain spectroscopy (THz TDS) is employed to measure the changes in the temporal profiles of the $\mathrm{THz}$ pulses transmitted through the graphene plasmonic cavities. For the generation of broadband $\mathrm{THz}$ waves, a mode-locked erbium-doped femtosecond fiber laser with a pulse repetition rate of $80 \mathrm{MHz}$ is used. The pulse width is approximately 80 fs (full width at half maximum), and the central wavelength is $1550 \mathrm{~nm}$.

The pulsed laser beam is focused onto a biased, lowtemperature-grown InGaAs/InAlAs $\mathrm{THz}$ photoconductive antenna (TERA15-TX, Menlosystems). The antenna has a gap spacing of $100 \mu \mathrm{m}$, and a bias of $15 \mathrm{~V}$ is applied with an average optical power of $20 \mathrm{~mW}$. The linearly polarized $\mathrm{THz}$ wave emitted by the antenna is collected and refocused onto the sample from a $45^{\circ}$ oblique angle (and subsequently onto the $\mathrm{THz}$ detector) using off-axis parabolic mirrors. A small $250-\mu \mathrm{m}$-diameter hole at the end of a tapered aperture of a conical shape is placed close to the sample. We check that this aperture is not destroying the polarization of the incoming beam. $\mathrm{THz}$ radiation is detected using a second low-temperature-grown InGaAs/InAlAs THz photoconductive antenna made of the $25 \mu \mathrm{m}$ dipole and $10 \mu \mathrm{m}$ gap excited with an average optical power of $17 \mathrm{~mW}$ (at $1550 \mathrm{~nm}$ ).

All the TDS measurement are performed in a time window of 12 ps with a temporal resolution of $6.67 \mathrm{fs}$. Examples of typical time traces and spectra obtained with and without electrical doping are shown in Fig. 3(c) as orange and green lines, respectively. The time traces in the time window of $12 \mathrm{ps}$ are used in the calculation of the Fourier transform spectra. Typical Fourier spectra [obtained from Fig. 3(c)] in a linear scale are shown in Fig. 3(d), confirming an effective frequency bandwidth going from $0.1 \mathrm{THz}$ up to above $4 \mathrm{THz}$ with a rather high signal-tonoise ratio (above $10^{4}$ ) in the whole range. In the rest of the work, we restrict the presentation for time and frequency ranges where the real differences between the measurement with and without electrical doping (with and without strong positive gate polarization) are well seen. The sample stage 


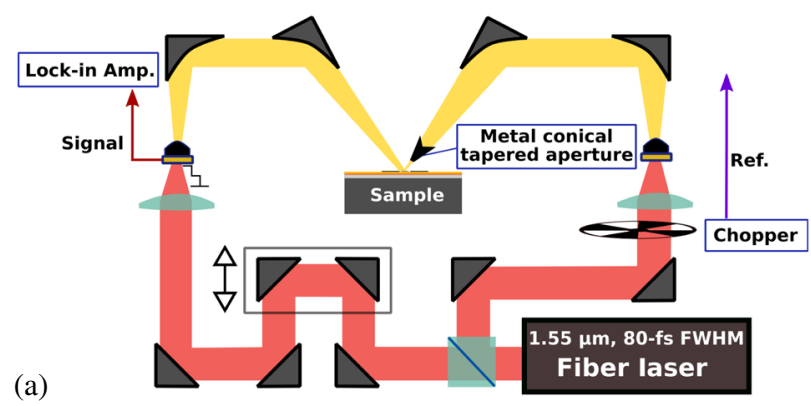

(b)
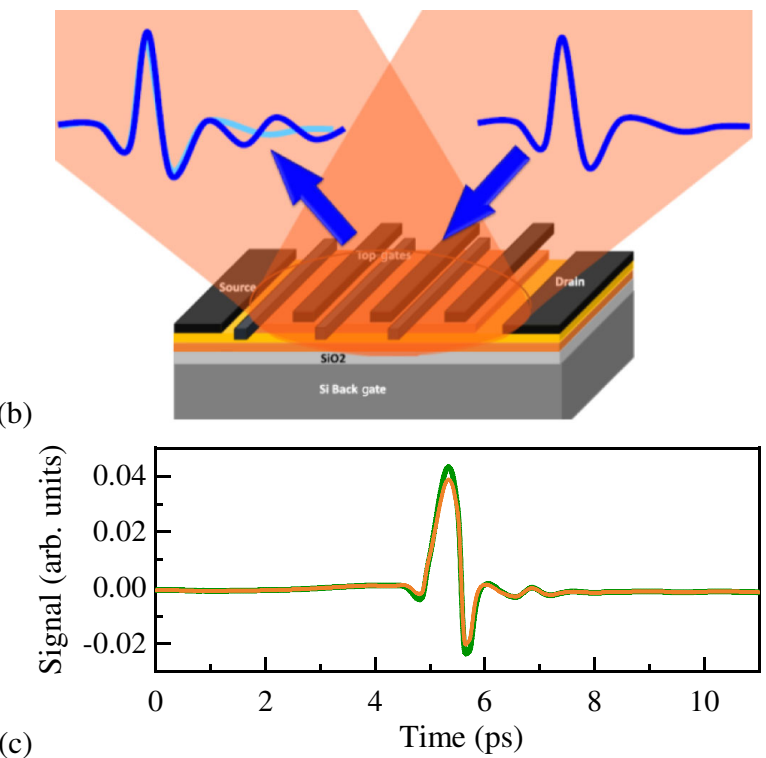

(c)

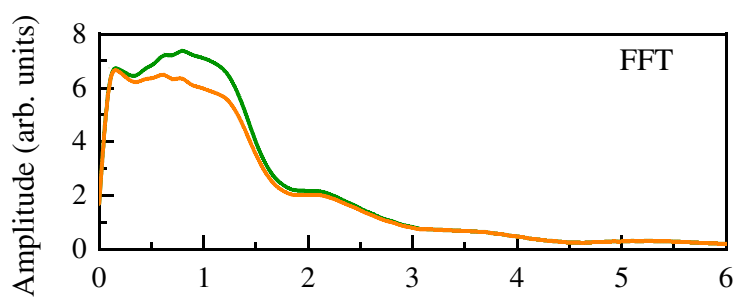

(d)

Frequency (THz)

FIG. 3. Measurement setup. (a) THz TDS. (b) Total reflection configuration. The incident $\mathrm{THz}$ radiation pulse is linearly polarized. The sample stage is rotated to obtain the radiation polarization perpendicular or parallel to the drain-source direction. (c) Typical time trace for samples with (orange) and without gate biasing (green) in the measurement system are shown in the upper panel. Spectra are obtained by FFT transformation of the same signal in the lower panel.

is rotated to align the $\mathrm{THz}$ radiation polarization perpendicular or parallel to the grating finger direction.

In the first part of the experimental studies, we measure the spectra for zero drain-to-source voltage $\left(U_{d}=0\right)$. As mentioned above, the spectra are always recorded with only one gate voltage biased away from the CNP and with all other gates biased at the CNP. In the second part of the experimental studies, $U_{d}$-dependent measurements are performed.
We use the reflection configuration, as shown in Fig. 3(b). The $\mathrm{THz}$ pulse is transmitted twice through the $h$-BN, graphene, and thin $h-\mathrm{BN} / \mathrm{SiO}_{2}$ dielectric layers. Because of highly doped low-resistivity conditions of the Si substrate with the $45^{\circ}$ oblique angle incidence, the interface gives total reflection. When the THz pulse travels through such a path, it reflects and transmits through at the interfaces between graphene and the $h$-BN buffer layer and between the $h$-BN buffer layer and the $\mathrm{SiO}_{2}$ layer.

Such a multilayered vertical structure makes a superposition of the reflected pulses at each interface in its temporal pulse profile. Since the thicknesses of the $h$-BN buffer layer (approximately $40 \mathrm{~nm}$ ) and $\mathrm{SiO}_{2}$ layer (approximately $90 \mathrm{~nm}$ ) are far smaller than the wavelength of the $\mathrm{THz}$ radiation, the artifacts and distortions caused by the multiple reflections on the temporal pulse profile are negligibly small (the round-trip delay time from or to the top graphene surface is approximately $3 \mathrm{fs}$, which is almost 2 orders shorter than the $\mathrm{THz}$ pulse width). Therefore, what is measured by the TDS is the total pulse after its double passage throughout the aforementioned multilayered sample.

Some typical representative data are shown in Fig. 4. In each measurement, we register two time traces and calculated spectra: (i) the first $T_{\mathrm{CNP}}^{2}$, with all gates biased the way ensuring the CNP condition of the whole graphene channel, and (ii) the second $T_{P}^{2}$, with only one type of grating gate "strongly biased" (up to $U_{g}-U_{\mathrm{CNP}} \sim 3 \mathrm{~V}$ ) while keeping all other parts of the sample at the CNP condition. Then we calculate $2 \tilde{A}=\left(1-T_{P}^{2} / T_{\text {CNP }}^{2}\right)$. As shown later, $2 \tilde{A}$ contains mainly information about the extinction. The left panels in Fig. 4 are examples of some data (time traces, Fourier transform, and resulting extinction) obtained without any drain-source biasing $\left(U_{d}=0\right)$, whereas the right panels are the results obtained with a rather strong drainsource biasing at $U_{d}=700 \mathrm{mV}$. Even if the differences in traces are difficult to see at first glance, they are very well resolved in the experiment because of the (aforementioned) very high signal-to-noise ratio (above $10^{4}$ in the whole presented spectral range). The shadowed regions in Fig. 4 are magnified in the neighbor panels to show the regions where the differences can be observed. First, one can clearly see that the time traces (and spectra) without and with strong biasing (electrical doping) show some wellresolved experimental differences. Second, one can observe that the application of additional drain-source biasing $(700 \mathrm{mV}$ ) inverses the order of the blue "without carriers" and red with carriers traces suggesting "negative transmission." This very important point is systematically investigated and discussed in the next parts of this paper.

Generally, the experimental spectra $T_{P}^{2}$ and $T_{\text {CNP }}^{2}$ contain information about $\mathrm{THz}$ transmission, reflection, and absorption. The key parameter that governs the underlying physics in an array of conducting strips is the ratio of the conductivity in the active region to the light velocity: 

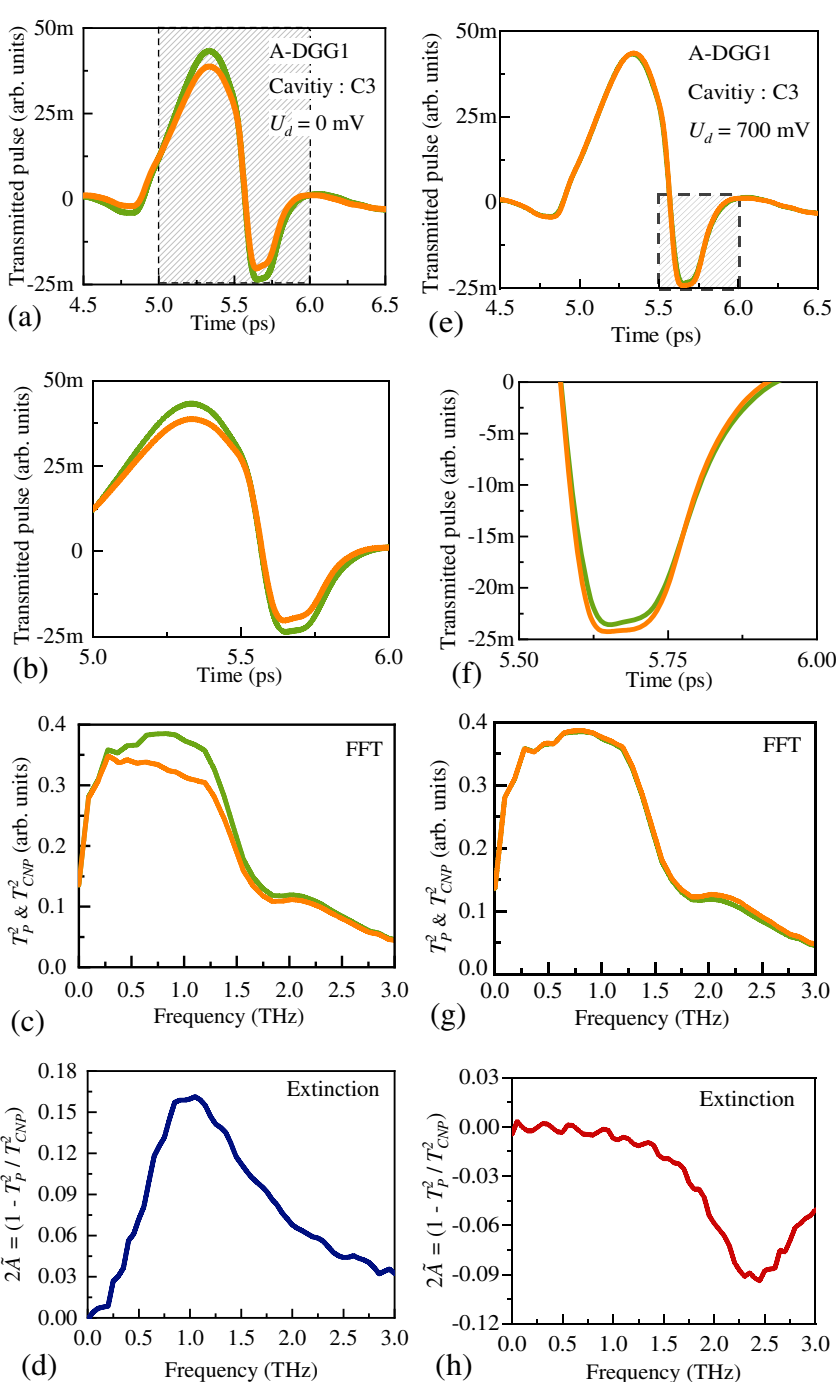

(g)

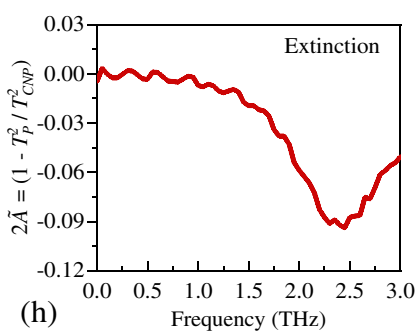

FIG. 4. Typical time traces obtained for the A-DGG1 sample with (orange) and without gate biasing (green). Left panels (a)-(c) are experiments without drain-source biasing $\left(U_{d}=0\right)$, and right panels $(\mathrm{e})-(\mathrm{g})$ the results obtained with a strong drain biasing at $U_{d}=700 \mathrm{mV}$. (b) and (f) are a magnification of the shadowed regions in (a) and (e), respectively. One can observe that the drain-source biasing inverses the order of magnitude of orange and green traces in the time traces and spectra row data. (d) and (h) show spectra obtained by Fourier transform for time traces with and without gate biasing, respectively.

$2 \pi \sigma_{1} / c \sqrt{\epsilon}$ (see Refs. [49,50], and the discussion in Sec. A of Supplemental Material [51]). Here, $\epsilon$ is the dielectric constant of the surrounding media.

For the case when this parameter is small,

$$
\frac{2 \pi \sigma_{1}}{c \sqrt{\epsilon}} \ll 1
$$

the transmission coefficient is approximately given by

$$
T \approx 1-A \text {, }
$$

where $A \ll 1$ is the absorption coefficient (see Supplemental Material [51]). The quantity, which is measured in the experiment, is proportional to $T^{2}$, because the incoming beam goes through the array of the conducting strips, reflects from the metallic substrate (mirror), and goes again through the array of the conducting strips. We get

$$
T^{2} \approx(1-A)^{2} \approx 1-2 A .
$$

As mentioned above, the quantity that we interpret in the experiment is $\left(1-T_{P}^{2} / T_{\mathrm{CNP}}^{2}\right)$, where $T_{\mathrm{CNP}}^{2}$ is the spectra registered with the whole sample at the CNP condition, and $T_{P}^{2}$ is the spectra obtained with only one of the grating gates "biased." Using Eq. (3), one can show that the measured quantity is proportional to $2 A$. In fact, the correction to the transmission coefficient in our experiment is smaller, because the size of the beam $S_{b}$ is bigger than the sample grating area $S_{g}$. The measured absorption is given by $2 \tilde{A}=2 A S_{b} / S_{g}$.

The coefficient $A$ can be simply expressed in terms of a radiation-induced correction to the dissipation in the system, $\delta P$ :

$$
A=\frac{\delta P}{\mathcal{S}}=\frac{8 \pi \delta P}{c \sqrt{\epsilon} E_{0}^{2}} .
$$

Here, $E_{0}$ is the amplitude of the incoming radiation, and $\mathcal{S}=c \sqrt{\epsilon} E_{0}^{2} / 8 \pi$ is the time-averaged radiation Pointing vector for a linearly polarized wave. Using Eqs. (3) and (4), we obtain $\left(1-T_{P}^{2} / T_{\mathrm{CNP}}^{2}\right) \approx 2 \tilde{A} \propto \delta P$, which is the quantity $2 \tilde{A}$ that we plot in all experimental figures. Importantly, this quantity is proportional to the dissipation in the channelthe property which we use for the theoretical interpretation of our results.

We emphasize that, in addition to dissipation in the channel, there also exist radiation losses. However, a detailed analysis performed in Ref. [49] for a similar structure shows that the radiation losses are small when condition (1) is satisfied. In particular, the rate of radiation attenuation ( $\Gamma$ in the notation of Ref. [49]) is small compared to the momentum relaxation rate $\gamma$ provided that the condition (1) is fulfilled. Therefore, it is enough for us to analyze the rate of dissipation in the channel, which radically simplifies the calculations.

\section{EXPERIMENTAL RESULTS}

\section{A. Resonant plasmons at zero drain current conditions}

In Fig. 5, we show the results of some systematic measurements with zero drain voltage or current applied to the structures $\left(U_{d}=0 \mathrm{~V}\right)$.

A comparison of the measurement results for two different polarizations of the $\mathrm{THz}$ radiation, parallel and perpendicular to the gate fingers, is shown in Figs. 5(a) and 5(b), respectively. This comparison is done for the 

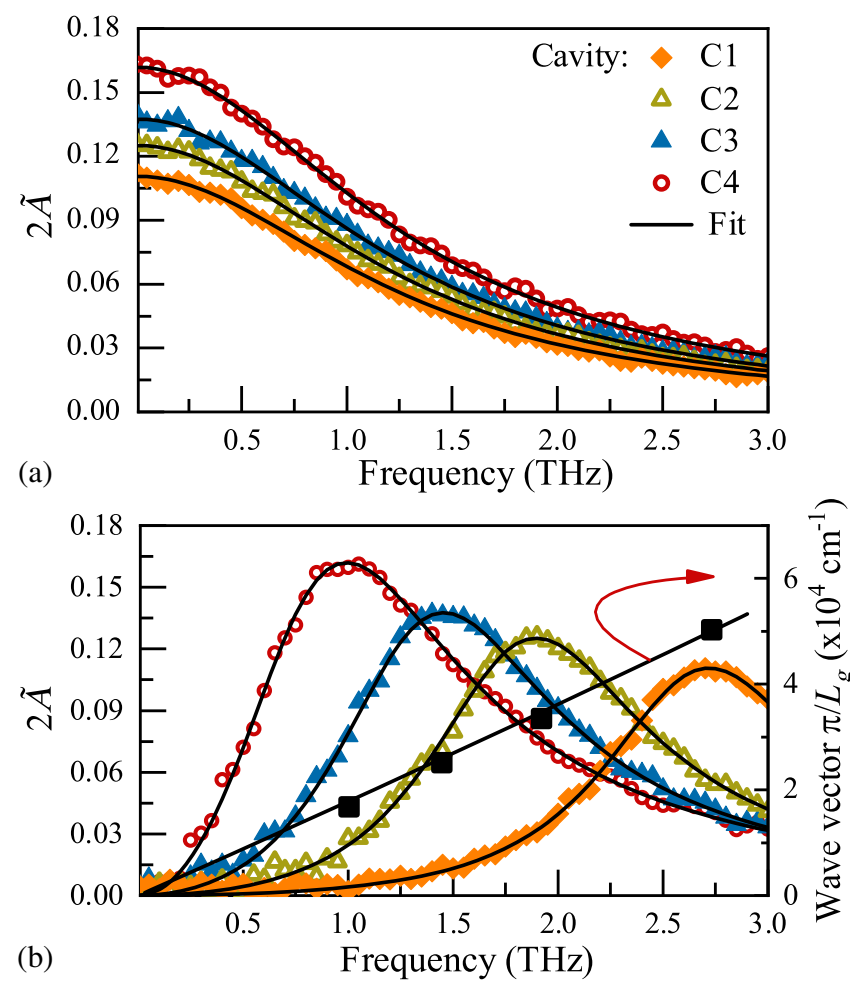

FIG. 5. Gate-length-dependent extinction spectra of the graphene structures for $U_{d}=0 \mathrm{~V}$ with incident light polarized parallel (a) and perpendicular (b) to the gate fingers. The measured data points and lines are the fits by the Drude model fit for parallel polarization and the damped oscillator model for perpendicular polarization. Black solid squares are a maximum of experimental resonant plasma frequencies as a function of the wave vector $q=\pi / L_{1}$. The black straight line through these dots is the best linear fit (right-hand scale).

same electrical doping of cavities $\left(U_{g}-U_{\mathrm{CNP}} \sim 3 \mathrm{~V}\right)$. One can see that, in the parallel polarization case, the extinction spectra are characterized by the Drude-like response with a monotonic decrease of the absorption with frequency [50]. In contrast, in the perpendicular polarization, a completely different line shape is observed with a pronounced resonant absorption peak shifting to higher energies for narrower gate fingers.

In the case of the polarization parallel to the stripes, the dissipation is due to the Drude absorption, and measured extinction spectra can be fitted by using the Drude formula:

$$
\tilde{A} \propto \frac{\gamma_{1}}{\gamma_{1}^{2}+\omega^{2}},
$$

where $\gamma_{1}=1 / \tau_{1}$ is the plasmon relaxation rate in the active area and the coefficient in this equation is frequency independent. The fitted values of the amplitude and plasma frequency vary for each cavity, while the variation of $\tau_{1}$ is fairly small: $0.12-0.13 \mathrm{ps}$.

Likewise, using the results of Supplemental Material [51], we fit the frequency dependencies of the extinction spectra for the perpendicular polarization case by the formula of the so-called damped oscillator model [50,52-54]:

$$
\tilde{A} \propto \frac{\gamma_{1}}{\gamma_{1}^{2}+\left(\omega-\omega_{1}^{2} / \omega\right)^{2}} \approx \frac{\gamma_{1} / 4}{\delta \omega^{2}+\gamma_{1}^{2} / 4},
$$

where $\omega_{1}$ is the resonance frequency in the active region with high conductivity and $\delta \omega=\omega-\omega_{1}$. The lines in Fig. 5 correspond to theoretical calculations. The calculated curves agree quantitatively very well with the extinction spectra registered in the experiments.

It is worth noting that the radiation with parallel polarization does not lead to a redistribution of charge even in the case of a nonzero dc current, so that plasmonic effects do not show up for such a polarization.

In Fig. 5(b), we show also the frequency as a function of the wave vector $\left(q=\pi / L_{1}\right)$, where $L_{1}$ is the single finger gate width of the active region with high conductivity (right-hand scale). One can see that the resonant frequency scales almost linearly with $q$ determined by the length of the grating finger, suggesting that in the investigated structures the grating fingers act as independent cavities with 2D gated plasmons with a dispersion relation close to $\omega=s_{1} q$, with $s_{1} \sim 3 \times 10^{6} \mathrm{~m} / \mathrm{s}$. The important thing is that plasma wave velocities are in all regions higher than the Fermi velocity in graphene. As a consequence, in our experimental situation, the carrier drift velocities in all regions are smaller than the plasma wave velocities.

Summarizing, the results from Fig. 5 show that (i) the metallic gates are close enough to 2DEG to grant dispersion very close to linear-strongly gated plasmon dispersionand (ii) it is not the grating period but the single finger dimension that determines the plasmon wave vectors.

In Fig. 6, we show the results of the study of the plasmon resonances, as a function of the gate voltage (2D carrier density). As in all experiments, the gate-voltage-dependent extinction spectra are shown while tuning only one cavity gate at a time and keeping all other parts of the sample at the charge neutrality condition. The gate-voltagedependent data show a clear blueshift and increase of the strength of the absorption peak frequency with increasing carrier density (increasing gate voltage). As shown in Figs. 6(a)-6(d), the fitting curves obtained using the damped oscillator model agree very well with the measured extinction spectra.

Figure 7 shows the gate-voltage dependences of the extracted plasmon frequencies. One can see that a sample with a shorter cavity length has a higher frequency at a given gate voltage. Figure 7 also shows theoretical plasmon frequencies, as functions of the gate voltage calculated as

$$
\omega_{q}=\sqrt{\frac{4 e^{2} T \ln \left[2+2 \cosh \left(\varepsilon_{F} / T\right)\right]}{\hbar^{2} \varepsilon q[1+\operatorname{coth}(q d)]}} q,
$$



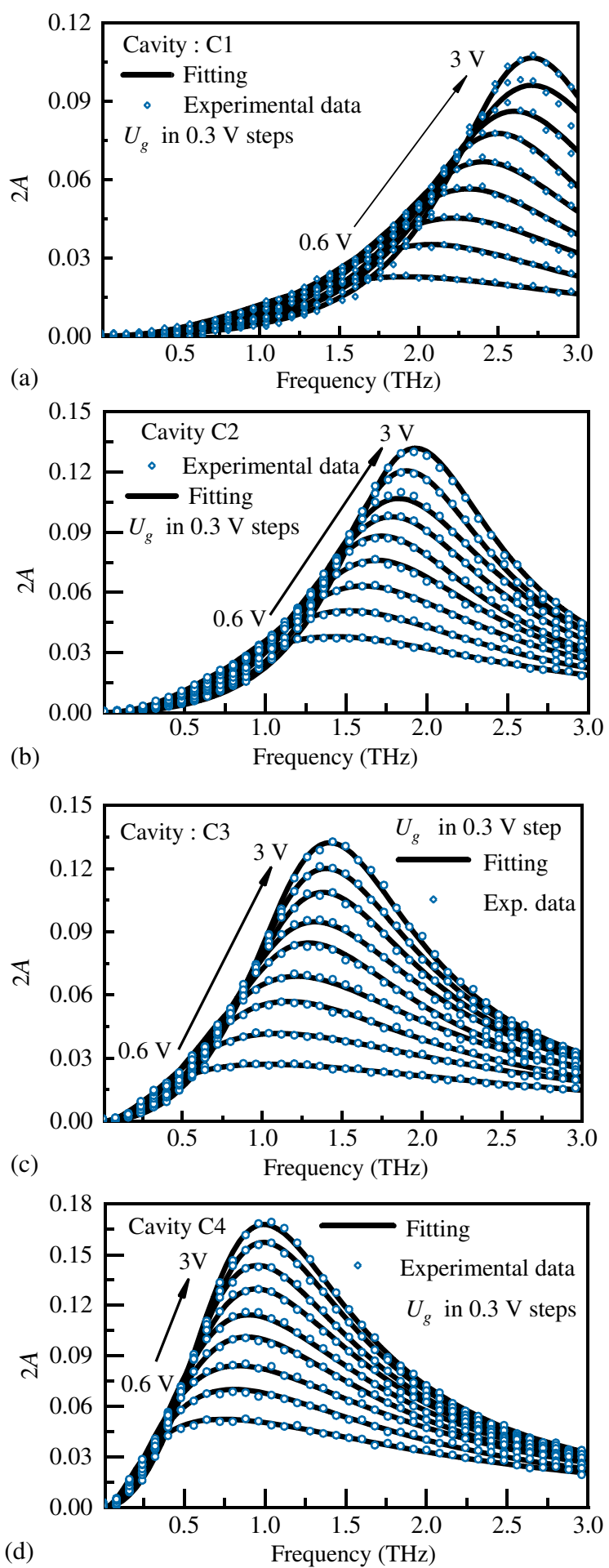

FIG. 6. Gate-voltage-dependent extinction spectra of the graphene structures for $U_{d}=0 \mathrm{~V}$ with incident light polarized perpendicular to the gate fingers. Panels (a), (b), (c), and (d) presents results for $C 1, C 2, C 3$, and $C 4$ cavities, correspondingly. The experimental data are marked as dots, and continuous lines are the results of fits using standard physical models of gated 2D plasmons in graphene.

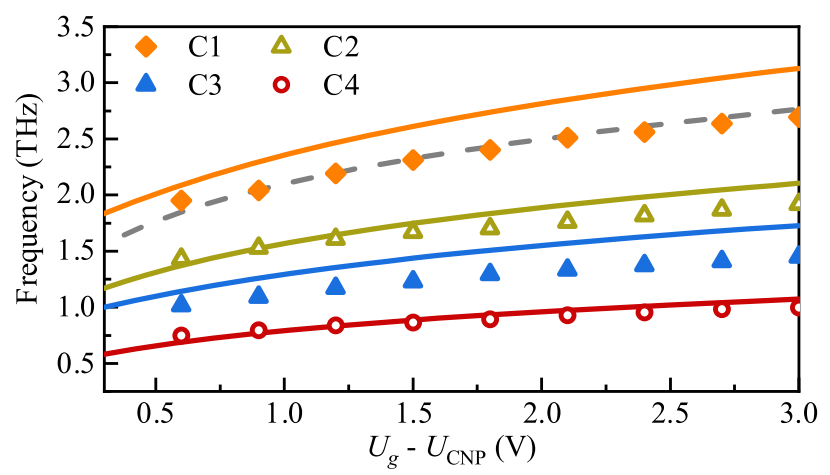

FIG. 7. Gate-voltage dependence of the extracted resonance frequency. Continuous lines are results of calculations according Eq. (7) with corrections to fringing and plasma leakage phenomena-see Supplemental Material [51] for more details. The dotted line is $1 / 4$ power dependence as a function of the gate bias, typical for plasmons in graphene.

where $q$ is the plasma wave vector, $d$ is the thickness of the top $h$-BN layer given in Table I, $\varepsilon=4.5$ is the dielectric constant of $h$-BN layers [55], $\varepsilon_{F}$ is the Fermi energy, and $T=300 \mathrm{~K}$ is the temperature. At a sufficiently high electron concentration $\left(\varepsilon_{F} \gg T\right)$, Eq. (7) simplifies to the well-known form [27,29]

$$
\omega_{q}=\frac{s q}{\sqrt{q d[1+\operatorname{coth}(q d)]}} .
$$

Here,

$$
s=\sqrt{\frac{4 e^{2} \varepsilon_{F} d}{\varepsilon \hbar^{2}}}
$$

is the plasma wave velocity ( $s=s_{1}$ in the active region, and $s=s_{2}$ in the passive region). The factor $q d[1+\operatorname{coth}(q d)]$ describes the deviation from the linear dispersion and is important when the thickness of the top $h$-BN layer is comparable with the gate lengths $\left(L_{1}\right.$ or $\left.L_{2}\right)$.

In the calculations, we take into account the quantum capacitance correction [48], fringing, and plasma leakage as predicted by the phenomenological model described later in the work and in Supplemental Material [51]. One can see that the theoretical calculations made without any fitting parameters reproduce relatively well the absolute values and the functional gate-voltage dependencies of the plasma frequency for all four cavities, close to $1 / 4$ power dependence. The small discrepancies may result from the uncertainty of $h$-BN layer thickness determination (precision of approximately 10\%) and unknown exact value of the dielectric function. In fact, in the literature, one can find $\epsilon$ in the range from 3.5 to 5 [55-58]. Also, in our calculations, the graphene Fermi velocity is assumed carrier density independent, where, in principle, it may change in 
the range between $1.3 \times 10^{6}$ and $1.0 \times 10^{6} \mathrm{~m} / \mathrm{s}$ in our experimental conditions $[58,59]$.

It should be stressed, however, that, independently of small numerical discrepancies, the observed plasma frequency follows $1 / 4$ power dependence on gate voltage (carrier density) typical for plasmons in graphene-as shown by the dotted line in Fig. 7 [see also Fig. S6(b) in Supplemental Material [51]].

$\mathrm{THz}$ plasmon resonances in graphene are already observed in microribbons $[50,60,61]$, rings, disks [52,53, 62,63], and grating-gate structures [38]. However, it is worth mentioning that (i) here, we report the first experimental observations of such plasma standing waves with $100 \%$ gate tunable frequency and (ii) the plasmon-related extinction coefficient is going up $19 \%$.

To summarize this part, the scaling behavior of plasmon frequency versus the plasmon wave vector and the gate voltage shown in Figs. 5-7 are successfully calculated using standard physical models of gated 2D plasmons in graphene [27-29].

They clearly confirm the existence of $2 \mathrm{D}$ plasmons oscillating at $\mathrm{THz}$ frequencies in our graphene $/ h-\mathrm{BN}$ nanostructures and allow an unambiguous attribution of the observed resonances to $2 \mathrm{D}$ plasmons in the individual fingers of the grating-gate defined cavities $C 1, \ldots, C 4$.

\section{B. Plasma resonances in the presence of dc current}

The most important part of the present work concerns the experimental investigations of the influence of the dc drain-source bias $U_{d}$ on the $\mathrm{THz} 2 \mathrm{D}$ plasmon resonances identified earlier.

In Fig. 4, one can see examples of experimental time traces and their Fourier transform for cavity $C 2(1 \mu \mathrm{m})$ with and without applied drain bias. The results, first $\left(T_{\mathrm{CNP}}^{2}\right)$ with all the gates "unbiased" (set at the CNP) and second with only the $C 2$ cavity gate "biased" at approximately $3 \mathrm{~V}$ $\left(T_{P}^{2}\right)$, are plotted. Comparing the results with and without drain-source biases, one can clearly see that in the range of plasmonic resonances the order of the traces with and without gate biasing is inverted after switching on the drain-source voltage $(700 \mathrm{mV})$. First, for zero drain bias, when cavity $C 2$ is filled with carriers, the outgoing light intensity is lower than the incoming one (plasma resonant absorption). For high drain voltage $(700 \mathrm{mV})$, in contrast, the registered signal is higher when the cavity is filled with carriers (amplification or emission). This result is the reason why in the last right panels data plotted as $2 \tilde{A}$ show negative values.

To further investigate this intriguing behavior, we perform very systematic measurements versus the drain bias for all four cavities. In Fig. 8, we show a 3D plot illustrating the typical drain bias dependence of the extinction $(C 2$ plasma cavity). With an increase of the dc current, a strong redshift of the resonant $\mathrm{THz}$ plasmon absorption, a region of complete transparency to incoming radiation, followed

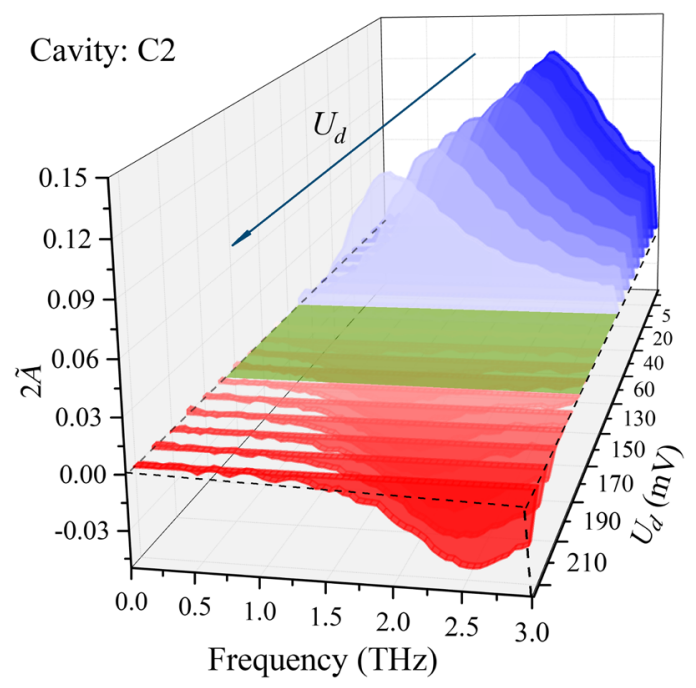

FIG. 8. Drain-bias-dependent spectra for the $C 2$ plasma cavity. Blue marks absorption, the green zone is the gap between absorption and emission, and red corresponds to amplification. The arrow indicates raising drain bias.

by the amplification and blueshift of the resonant plasmon frequency is observed.

In Fig. 9, we collect all results in the form of the 2D plots-keeping the color code as in Fig. 8. As $U_{d}$ increases, the absorption peak clearly shifts to lower frequencies along with a noticeable reduction of plasmon resonance amplitude. Then, the absorption completely vanishes, as seen, for example, in the measured extinction spectra for cavity $C 1$, being zero at $U_{d} \sim 45 \mathrm{mV}$ up to $U_{d} \sim$ $90 \mathrm{mV}$ [see Fig. 9(a)]. In this drain voltage range, the plasmonic device $C 1$ becomes perfectly transparent to the incoming $\mathrm{THz}$ radiation within the entire experimental bandwidth. It is important to stress that, here, we report the first experimental observation of such a strong Doppler shift of plasma resonances in graphene and the transparency behavior over a relatively wide frequency range $(0.1-3 \mathrm{THz})$. With increasing $U_{d}$ beyond this transparency regime, a resonant "negative absorption" feature appears in the extinction spectra and shifts with increasing drain bias toward higher frequencies (noticeable blueshift).

Throughout all of our experiments, the data from all cavities $C 1, \ldots, C 4$ are quite similar with the amplification threshold voltage increasing with the cavity length $L_{g}$ and important widening of the transparency range (from about 50 up to $300 \mathrm{mV}$ ).

The results presented in Fig. 9 show some universal behavior. This behavior is visualized when we present them in the rescaled mode. In Fig. 10, the vertical axis is the frequency divided by its value at $U_{d}=0$, and the drain voltage (the horizontal axis) is normalized by the drain voltage $U_{0}$ at which the plasmon frequency tends to zero (see Fig. 9). Very good quantitative agreement both at low 

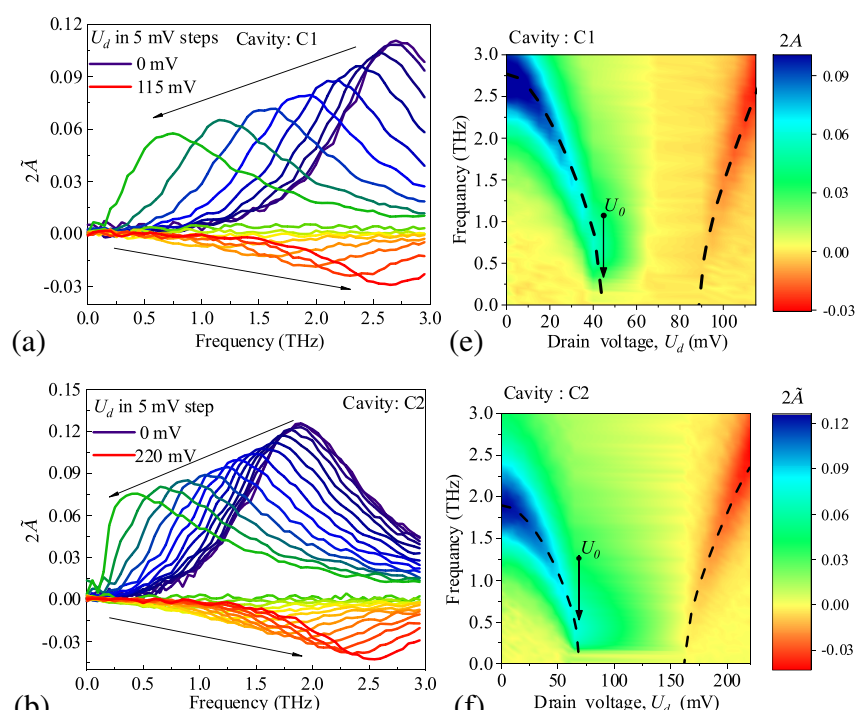

(b)
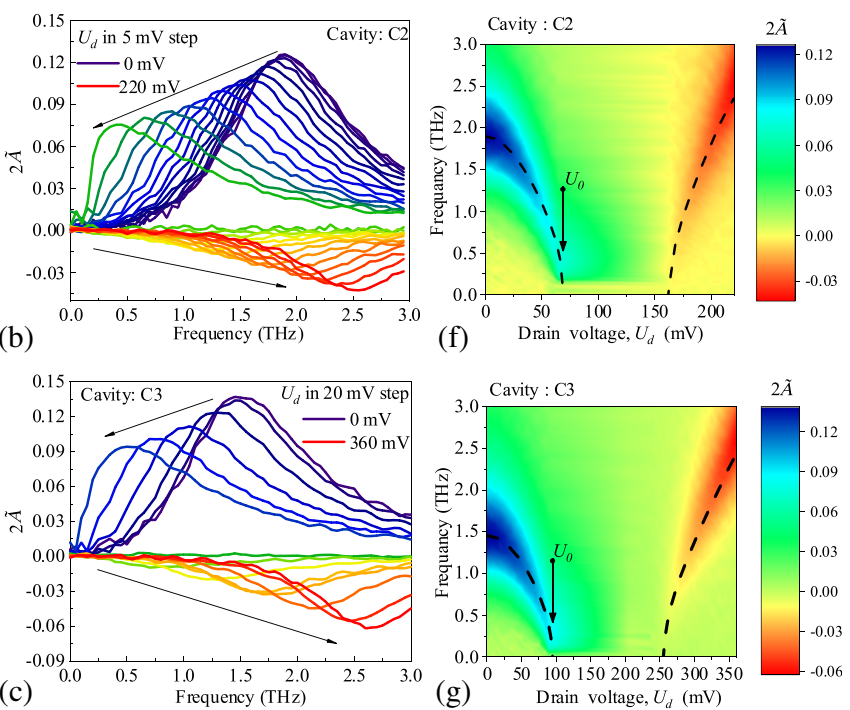

(f) Drain voltage, $U_{d}(\mathrm{mV})$
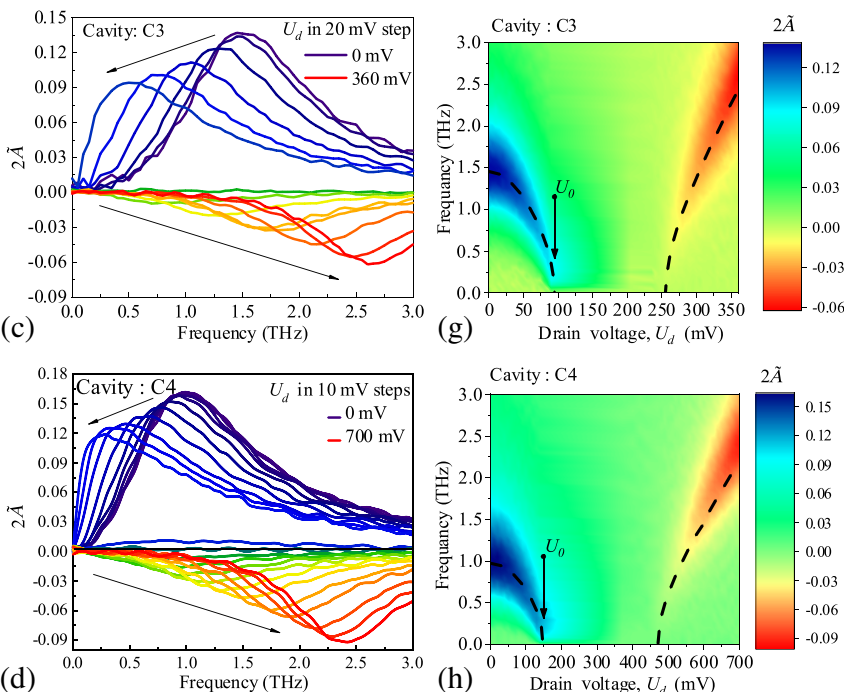

FIG. 9. Drain-bias-dependent properties of the $C 1, C 2, C 3$, and $C 4$ plasma cavities. The left-hand panels show the spectra (a), (b), (c), and (d), correspondingly. The right-hand panels show the contour plots (e), (f), (g), (h), correspondingly. The dotted lines on contour plots are results of the fit with a phenomenological formula Eq. (10).

and at high currents can be obtained by fitting experimental curves as

$$
\frac{\omega_{1}^{2}(x)}{\omega_{1}^{2}(0)}=1+\zeta+x^{2}-\sqrt{\zeta^{2}+4 x^{2}(1+\zeta)},
$$

where $\omega_{1}(x)$ stands for the position of the plasmonic resonance, $x=U_{d} / U_{0}$, and $\omega_{1}(0)$ is the experimentally measured value of the plasmonic frequency at zero current. $U_{d}$ is the voltage across the structure, $U_{0}$ is the voltage corresponding to the onset of the transparency window, and $\zeta$ is a fitting parameter. The values of $\zeta$ corresponding to solid lines in Fig. 10 are presented in Table II. The unified universal behavior of frequency at low currents (for $x<1$ )

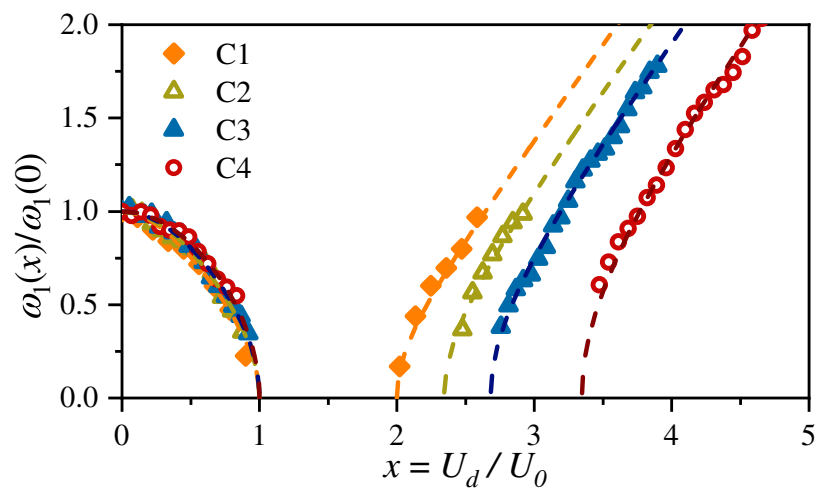

FIG. 10. The experimental results showing the resonant frequency versus drain voltage bias in normalized scales. Dotted lines show results of calculations according to Eq. (10).

can be obtained by taking in Eq. (10) the limit $\zeta \rightarrow \infty$, which yields

$$
\frac{\omega_{1}^{2}(x)}{\omega_{1}^{2}(0)}=1-x^{2}=1-\left(\frac{U_{d}}{U_{0}}\right)^{2} .
$$

The similar functional dependence of the plasmon frequency versus electron drift velocity of $2 \mathrm{D}$ electron gas in $\mathrm{GaAs} / \mathrm{AlGaAs}$ grating-gate structures [Eq. (10)] is obtained in Ref. [49]. However, the physical model used in this work cannot be applied to interpret our results. The validity of the physical model of Ref. [49] for our structures and analogy used to derive Eq. (10) is discussed in more detail in the following section and in Supplemental Material [51].

\section{PHENOMENOLOGICAL DESCRIPTION OF THE OBTAINED RESULTS}

\section{A. Plasmonic crystal model}

In phenomenological interpretation, we consider a plasmonic crystal composed of two different regions with lengths $L_{1}$ and $L_{2}$, plasma velocities $s_{1}$ and $s_{2}$,

TABLE II. Properties of different plasma cavities.

\begin{tabular}{lcccc}
\hline \hline Cavity name & $C 1$ & $C 2$ & $C 3$ & $C 4$ \\
\hline Structure or sample & A-DGG2 & A-DGG1 & A-DGG2 & A-DGG1 \\
$L_{1}(\mu \mathrm{m})$ & 0.5 & 0.75 & 1.0 & 1.5 \\
$L_{2}(\mu \mathrm{m})$ & 3.5 & 3.0 & 3.0 & 2.25 \\
$U_{0}(\mathrm{mV})$ & 45 & 69 & 92 & 143 \\
$U_{T}(\mathrm{mV})$ & 90 & 159 & 267 & 458 \\
$s_{1}\left(10^{8} \mathrm{~cm} / \mathrm{s}\right)$ & 3.2 & 3.2 & 3.1 & 3.1 \\
$s_{2}\left(10^{8} \mathrm{~cm} / \mathrm{s}\right)$ & 1.6 & 1.6 & 1.6 & 1.6 \\
$f_{1}=\omega_{1} / 2 \pi(\mathrm{THz})$ & 2.76 & 1.90 & 1.45 & 0.97 \\
$f_{2}=\omega_{2} / 2 \pi(\mathrm{THz})$ & 0.11 & 0.23 & 0.24 & 0.32 \\
$\zeta$ & 1.5 & 2.3 & 3.1 & 5.1 \\
$n_{1}\left(10^{12} \mathrm{~cm}^{-2}\right)$ & 2.0 & 2.0 & 2.0 & 2.0 \\
$n_{2}\left(10^{12} \mathrm{~cm}^{-2}\right)$ & 0.1 & 0.1 & 0.1 & 0.1 \\
\hline \hline
\end{tabular}




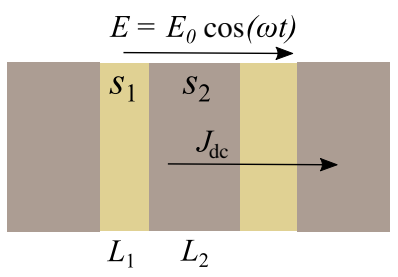

FIG. 11. 1D plasmonic crystal driven by dc current in the oscillating field of $\mathrm{THz}$ radiation.

carrier densities $n_{1}$ and $n_{2}$, and drift velocities $\mathcal{V}_{1}$ and $\mathcal{V}_{2}$ (see Fig. 11). We assume that $n_{1} \gg n_{2}$ and refer to region " 1 " as active and region " 2 " as passive. The plasma wave velocities $s_{1}$ in the electrically doped active cavities (for gate voltage $3 \mathrm{~V})$ are calculated from experimental data shown in Fig. 7, using Eq. (8). The results are shown in Table II. One can see that for all cavities $s_{1} \sim 3.2 \times 10^{8} \mathrm{~cm} / \mathrm{s}$. Because the plasma velocity is proportional to the $(1 / 4)$ power of the carrier density [see Eq. (9)], the plasma velocities in CNP passive cavities can be estimated as $s_{2} \sim s_{1} / 2 \sim 1.6 \times 10^{8} \mathrm{~cm} / \mathrm{s}$. The drift velocities in the electrically doped regions (active regions) $\mathcal{V}_{1}$ are by more than one order of magnitude lower than those in the CNP regions. The ratio $\left(\mathcal{V}_{2} / \mathcal{V}_{1} \sim n_{1} / n_{2} \sim 10\right)$ of the drift velocities stems from the dc current continuity condition $\left(n_{1} \mathcal{V}_{1}=n_{2} \mathcal{V}_{2}\right)$. We also take into account in this subsection that damping rates in the passive and active regions can be different. We assume, however, that they have the same order of magnitude: $\gamma_{1} \sim \gamma_{2}$.

Importantly, plasma wave velocities are in all regions higher than the Fermi velocity in graphene. As a consequence, in our experimental situation, the carrier drift velocities in all regions are smaller than the plasma wave velocities.

Therefore, the most important theoretical challenge is to find the physical process or mechanism in which the amplification can take place for drift velocities below the plasma wave velocity. In this work, we develop a phenomenological theory which shows that, indeed, amplification is possible for $\mathcal{V}_{1}<s_{1}$. This theory shows a possible way of physical interpretation and gives universal dependence of the plasmonic frequency on the current in a sense that the only controlling parameter is $\mathcal{V}_{1} / s_{1}$.

Let us consider the structure shown in Fig. 11. In the absence of dissipation, such a structure represents an example of 1D plasmonic crystal, with the plasma wave spectrum $\omega(k)$, which describes allowed and stop bands and should be found from the following dispersion equation [64]:

$\cos (k L)=\cos \left(\omega T_{1}\right) \cos \left(\omega T_{2}\right)-Z \sin \left(\omega T_{1}\right) \sin \left(\omega T_{2}\right)$.

Here, $k$ is the plasmon quasimomentum, $T_{1}=L_{1} / s_{1}$ and $T_{2}=L_{2} / s_{2}$ are the plasmon transit times, and

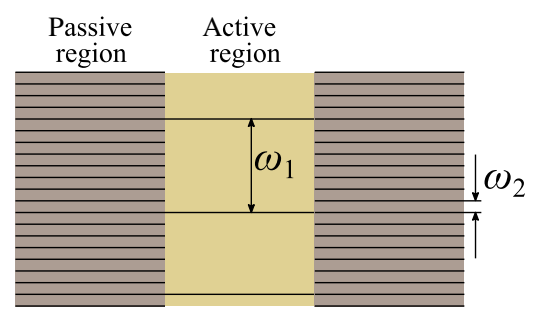

FIG. 12. Active regions of the plasmonic crystal with a large fundamental frequency are separated by passive regions with a small frequency.

$Z=\left(s_{1}^{2}+s_{2}^{2}\right) /\left(2 s_{1} s_{2}\right)$ is the mismatch parameter. For simplicity, we consider theoretically only the case $s_{1} \gg s_{2}$, when $Z \gg 1$. In this case, allowed bands have negligible widths and can be found from the equation $\sin \left(\omega T_{1}\right) \sin \left(\omega T_{2}\right)=0$, which describes plasmonic oscillations in the active regions with the fundamental frequency $\omega_{1}=\pi s_{1} / L_{1}$ and in the passive regions with the fundamental frequency $\omega_{2}=\pi s_{2} / L_{2}$ (see Fig. 12).

Let us assume that this structure is illuminated by radiation with the large wavelength, $\lambda \gg L_{1}, \lambda \gg L_{2}$, so that the electric field of radiation is approximately homogeneous. This field excites plasmonic oscillations in both active and passive regions. The plasmonic resonances occur when the frequency of the external field is equal to $n \omega_{1}$ and $m \omega_{2}$, where $n$ and $m$ are integer numbers. Equation (12) can be easily generalized for the realistic dissipative case $\gamma_{1} \neq 0, \gamma_{2} \neq 0$, when the spectrum of the plasmonic crystal acquires also an imaginary part: $\omega(k)=\omega^{\prime}(k)+i \omega^{\prime \prime}(k)$. The corresponding dispersion equation is quite cumbersome, and we do not present it here. However, the physical results obtained from this equation can be understood based on simple physical consideration.

We assume that

$$
s_{1} / L_{1} \gg \gamma_{1} \sim \gamma_{2} \gg s_{2} / L_{2} \text {. }
$$

In this case, resonances in the passive region strongly broaden and overlap. Physically, this result means that plasma waves rapidly decay along the passive region, which, in turn, implies that different active regions are disconnected at plasmonic frequencies. This result is in excellent agreement with experimentally observed independence of different active regions. On the other hand, different active regions are connected at zero dc frequency in the sense that dc current flows through the system.

Let us clarify this point in more detail. The oscillations decay into a passive region as $\propto \exp \left(-\gamma \delta x / s_{2}\right)$ (here, $\delta x$ is coordinate counted from the border between regions). Under the condition (13), this exponent at $\delta x=L_{2}$ becomes $\propto \exp \left(-\gamma L_{2} / s_{2}\right) \ll 1$. Hence, the coupling of oscillations in the neighboring active regions is exponentially small, and one can reduce the problem to the calculation of excitation in a single active strip with proper 
boundary conditions. A detailed discussion of such an approach is presented in Supplemental Material [51]. For zero current, the proper conditions look like

$$
\delta j_{1}(0)=\delta j_{1}\left(L_{1}\right)=0,
$$

which means that the current is fixed on both sides of the active region. Such conditions allow for a detailed quantitative description of plasmonic resonances in the transmission coefficient.

To check how inequalities Eq. (13) are fulfilled in our experimental situation, we have to estimate resonant frequencies in the passive and active plasma cavities. The length of CNP cavities does not vary much, and, for rough estimations, one can take $L_{2} \approx 3 \mu \mathrm{m}$, which leads to plasma angular frequency as $\omega_{2}=2 \pi f_{2} \approx 1.6 \mathrm{THz}$. One can see that the condition $\omega_{1} \gg \omega_{2}$ (or, equivalently, $\left.s_{1} / L_{1} \gg s_{2} / L_{2}\right)$ is relatively well fulfilled for all four experimental configurations (active cavities $C 1, \ldots, C 4$ ).

\section{B. Resonance frequencies and broadening of resonances for zero current}

Let us first consider the case of zero current. In this case, the existence of two plasma regions has two consequences - the plasma frequency is shifted toward lower frequencies and broadening appears (similar broadening is predicted for a single-gate problem in Ref. [65]). These two phenomena are described by the following equations (see Supplemental Material [51]):

$$
\gamma_{\mathrm{eff}}=\gamma_{1}+\frac{2 s_{1}}{L_{1}} \ln \left(\frac{s_{1}+s_{2}}{s_{1}-s_{2}}\right)
$$

where $\gamma_{1}=1 / \tau_{1}$, and $\tau_{1}$ is the momentum relaxation time in the active region [in this subsection, we assume that $\gamma_{1}$ can differ from $\gamma_{2}$ but have the same order of magnitude, $\gamma_{1} \sim \gamma_{2}$, so that inequality (13) holds for both $\gamma_{1}$ and $\gamma_{2}$ ]

$$
\delta \omega_{1}=-\frac{\pi\left(\gamma_{2}-\gamma_{1}\right) s_{1} s_{2}}{\left(s_{1}^{2}-s_{2}^{2}\right)\left[\pi^{2}+\ln ^{2}\left(\frac{s_{1}+s_{2}}{s_{1}-s_{2}}\right)\right]},
$$

The effective width of the plasma cavities $L_{1}$ should also include a correction related to the fringing effect that leads to the following correction of the plasmon frequency:

$$
\delta \omega_{1}^{\mathrm{fr}}=\frac{2 d}{L_{1}} \omega_{1}
$$

In Fig. 13, we show the momentum relaxation time $\tau_{1}$ determined from transport measurements together with plasmon relaxation time $\tau_{\text {eff }}=1 / \gamma_{\text {eff }}$, calculated using Eq. (15). We also show the experimental plasmon relaxation time determined from the fit of the resonances (see Fig. 6). One can see that the leakage-induced contribution to the damping reduces the relaxation time by

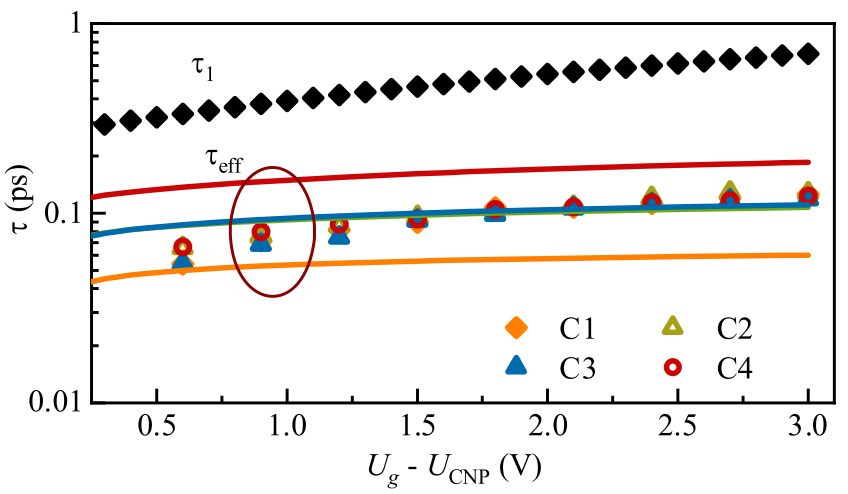

FIG. 13. Relaxation time as a function of the gate bias. $\tau_{1}$ is the momentum relaxation time in the active region shown as the black diamonds, $\tau_{\text {eff }}$ is the plasmon relaxation rate calculated using Eq. (15) (solid lines), and color dots are experimental $\tau_{\text {eff }}$.

approximately one order of magnitude, and calculated relaxation times are in relatively good agreement with experimental data.

This result explains why the widths of plasma resonances are much larger than those predicted from the transport experiments. It should be stressed that, in the plasmonic crystal approximation developed here, the correction to the resonant frequency [Eq. (16)] is relatively small. Therefore, the experiments in the absence of the drain bias can be interpreted by taking the standard graphene plasmon description of independent plasmon cavities Eqs. (7)-(9). For a more detailed discussion of plasmon resonance width and corrections to plasma frequency, see Supplemental Material [51].

\section{Energy dissipation and amplification}

Next, we discuss the most important experimental observation-the optical signal amplification observed when the drift velocity in the active region is smaller than corresponding plasma wave velocity $s_{1}$. To describe this phenomenon, we calculate dissipation in the channel under the drain biased condition and demonstrate that, with increasing the current, it decreases and changes sign, which implies amplification. We discuss the problem in the hydrodynamic regime, assuming that electron-electron collisions dominate over other types of scattering. (For a discussion of crossover from the hydrodynamic to the ballistic regime and of the Cherenkov instability in graphene, see Refs. [38,39].) The suggested mechanism should work for any electronic spectrum, but calculations are more compact and physically transparent for parabolic spectrum characterized by the effective mass $m$. We thus restrict ourselves to the discussion of this case only. Qualitatively, the results are valid also for graphene, where the role of $m$ is played by $E_{F} / v_{F}^{2}$.

The theoretical model that we develop in this work simplifies if, in addition to inequality (13), we also assume 
that $s_{1} \gg s_{2}$ [this inequality is different from Eq. (13) for $L_{2} \gg L_{1}$ ]. This condition is not strictly fulfilled (in our experimental situation, $s_{1} / s_{2} \sim 2$ only), and this result could be one of the possible reasons for the lack of quantitative agreement with experiment (see Sec. V and Supplemental Material [51]).

Since oscillations rapidly decay into passive regions, we consider dissipation in the active region only and put boundary conditions (14) for all active strips. Dissipation per unit area is given by

$$
P=\left\langle N \frac{m \mathbf{v}^{2}}{\tau}\right\rangle_{t, x},
$$

where $\tau$ is the momentum relaxation time, $N$ is the local concentration in the channel, and $\mathbf{v}$ is the total velocity given by the sum of the constant velocity related to the dc current and the oscillating component (see Ref. [66]). Equation (18) implies averaging over active and passive regions, where $N$ and $\tau$ have different values. However, the main contribution comes from region 1 , owing to the higher conductivity and resonant excitation. Hence, we skip the contribution of region 2 [here, in order to find dissipation averaged over the structure as a whole, the dissipation calculated in region 1 should be multiplied by the factor $\left.L_{1} /\left(L_{1}+L_{2}\right)\right]$.

The calculation of dissipation for the zero-current case is presented in Supplemental Material [51]. We derive there an exact formula for dissipation [Eq. (S21)] and demonstrate that it can be (with a good precision) replaced by a simplified equation obtained within the so-called damped oscillator model [see Eq. (S25) of Supplemental Material [51] ]. This equation, in turn, results in Eq. (6) for correction to the transmission coefficient. The latter equation perfectly fits the observed resonances provided that one replaces $\gamma_{1}$ with $\gamma_{\text {eff }}$. This fit gives additional confirmation of the plasmonic mechanism.

Let us now assume that we apply driving dc current in the system leading to nonzero electron drift velocity $\mathcal{V}_{1}$ in the active region. In this case, the expression for dissipation in the active region should be modified as follows:

$$
\begin{aligned}
P_{1} & =\left\langle N_{1}\left(1+n_{1}\right) \frac{m\left(\mathcal{V}_{1}+v_{1}\right)^{2}}{\tau_{1}}\right\rangle_{t, x} \\
& \approx \frac{m N_{1}}{\tau_{1}}\left(\mathcal{V}_{1}^{2}+\left\langle v_{1}^{2}+2 \mathcal{V}_{1} v_{1} n_{1}\right\rangle_{t, x}\right) .
\end{aligned}
$$

Here, $v_{1}$ and $n_{1}=\delta N_{1} / N_{1}$ are the radiation-induced corrections to the drift velocity and normalized concentration, respectively. Hence, radiation-induced correction to dissipation in the active region is given by

$$
\delta P_{1}=\frac{m N_{1}}{\tau_{1}}\left\langle v_{1}^{2}+2 \mathcal{V}_{1} v_{1} n_{1}\right\rangle_{t, x} .
$$

The second term in this equation arises due to the presence of the current. Remarkably, this term depends on the phase shift between $n_{1}$ and $v_{1}$ and can be negative. This result means that Eq. (20) is not positively defined, and for special cases $\delta P$ could become negative. This possibility implies switching from dissipation to amplification. The full theoretical description of the plasmonic crystal with two regions having arbitrary properties is quite cumbersome but can be essentially simplified for the case when damping of plasmonic oscillations in the passive region is sufficiently large, so that condition Eq. (13) holds.

We use the same approach as we use to study the response at zero current (see Supplemental Material [51]). Namely, we describe the problem phenomenologically, by using the same boundary conditions [Eq. (14)] as for the zero-current case.

We linearize the hydrodynamic equations and search for the solution in the following form: $v_{1}=\delta v_{1}(x) e^{-i \omega t}+$ H.c. and $n_{1}=\delta n_{1}(x) e^{-i \omega t}+$ H.c., where $\delta v_{1}(x)$ and $\delta n_{1}(x)$ obey

$$
\begin{gathered}
\left(\mathcal{V}_{1} \frac{\partial}{\partial x}-i \omega+\gamma_{1}\right) \delta v_{1}+s_{1}^{2} \frac{\partial \delta n_{1}}{\partial x}=\frac{F_{0}}{2 m}, \\
\left(\mathcal{V}_{1} \frac{\partial}{\partial x}-i \omega\right) \delta n_{1}+\frac{\partial \delta v_{1}}{\partial x}=0
\end{gathered}
$$

The oscillating correction to the current is given by $j_{1}=\delta j_{1}(x) e^{-i \omega t}+$ H.c., where

$$
\delta j_{1}(x)=s_{1}^{2}\left[\mathcal{V}_{1} \delta n_{1}(x)+\delta v_{1}(x)\right] .
$$

We solve Eqs. (21) and (22) with boundary conditions Eq. (14), substitute $v_{1}$ and $n_{1}$ into Eq. (20), and average over time and space. The solution thus obtained depends on the current, which is encoded in the drift velocity without radiation, $\mathcal{V}_{1}$. Calculations are very similar to the conventional calculation of the response of a single FET (see Refs. $[9,67])$. The dependence of total averaged dissipation $\delta P=\left[L_{1} /\left(L_{1}+L_{2}\right)\right] \delta P_{1}$ on $\omega$ for different $\mathcal{V}_{1} / s_{1}$ is shown in Fig. 14. Qualitatively, this dependence is similar to the experimentally observed one. Indeed, as seen with increasing the current, the frequency and the amplitude of the plasmonic resonance decrease. For a sufficiently large current, $\delta P$ changes sign, which implies amplification. It is worth noting, however, that there appear some new peaks in the amplification, which are not seen in the experiment. The general analytical expression for dissipation simplifies to the form allowing an analytical solution if the quality factor of resonance is high so that its frequency is much larger than damping. For this case, assuming $L_{1}=L_{2}=L$, we get

$$
\delta P=\frac{F_{0}^{2} N_{1}}{m \pi^{2}} \frac{\gamma_{\mathrm{eff}}}{\left[\omega-\omega_{1}(x)\right]^{2}+\gamma_{\mathrm{eff}}^{2} / 4^{2}} A(x),
$$




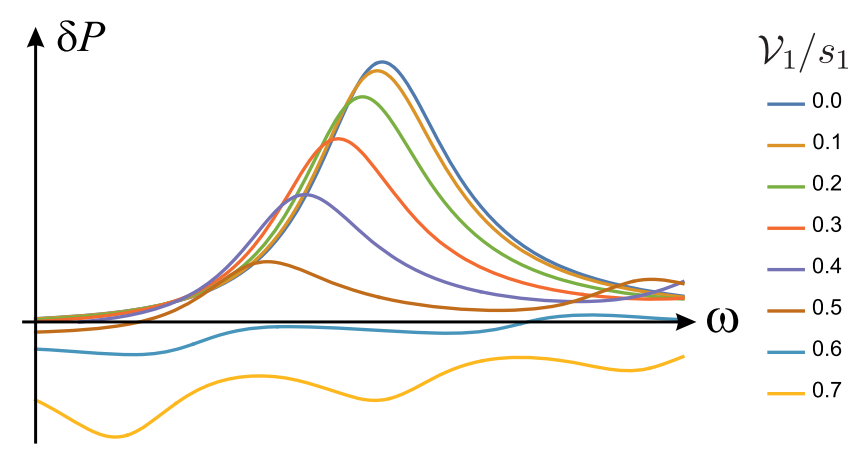

FIG. 14. Dissipation in the channel for different values of $\mathcal{V}_{1} / s_{1}$.

where

$$
\omega_{1}(x)=\frac{\pi s_{1}}{L_{1}}\left(1-x^{2}\right)
$$

and

$$
A(x)=\frac{\left(1-3 x^{2}\right) \cos ^{2}(\pi x / 2)}{\left(1-x^{2}\right)^{2}}
$$

are the frequency and amplitude of the resonance, respectively, which depend on $x=\mathcal{V}_{1} / s_{1}$. Even if this analytical solution is obtained for conditions somehow far from the experimental ones, it captures the main characteristics. It shows both the Doppler shift and the possibility of amplification. Indeed, the frequency $\omega_{1}(x)$ depends on the current [see Eq. (25)], while amplitude $A(x)$ turns to zero at a certain value of $x=x_{0}=1 / \sqrt{3}$, which is the dissipation-amplification transition point as shown in Fig. 15.

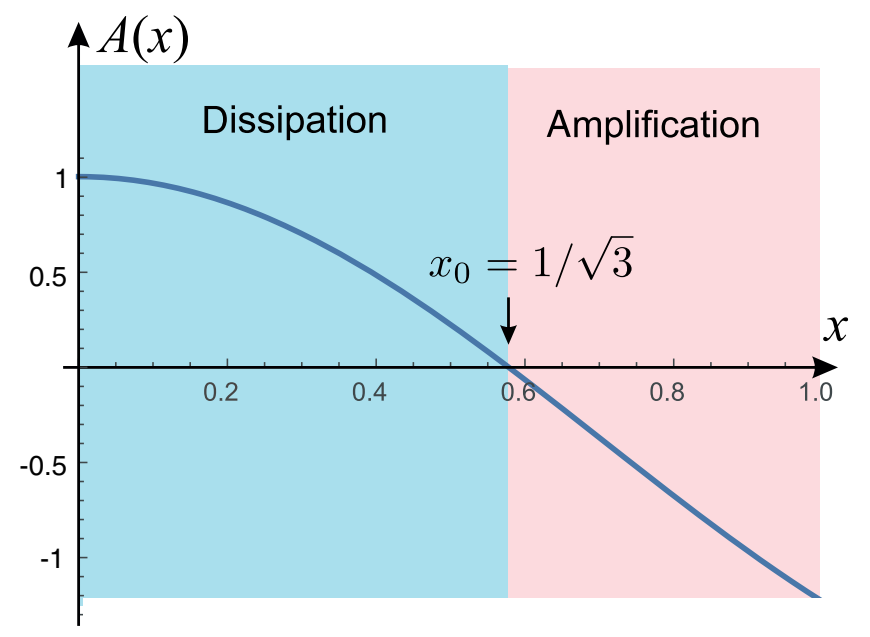

FIG. 15. The amplitude of the dissipation as a function of $x=\mathcal{V}_{1} / s_{1}$. Dissipation-amplification transition corresponds to $x=x_{0}=1 / \sqrt{3}$.

\section{DISCUSSION}

Let us briefly discuss other known mechanisms of amplification and instability.

First of all, it is instructive to compare our theoretical model with the theory developed in Ref. [49] for gratinggated 2DEG in GaAs. This theory predicts that with the increase of the current (drift velocity) one may observe a strong Doppler shift of plasma resonance followed by $100 \%$ transparency range and amplification. These predictions are very similar to our observations. Moreover, in principle, Eq. (10) can be obtained from Eq. (52) of Ref. [49] (see Supplemental Material [51]). However, despite the good description by Eq. (10), the physical interpretation using the models of Ref. [49] is not possible. This model describes a different physical situation; namely, in contrast to our system, the electron concentration is homogeneous in the absence of radiation.

The second reason, which is even more important, is the fact that to explain amplification within this model one uses Cherenkov-like effects that require drift velocity in the active region to be higher than the plasma wave velocity. Indeed, it turns out that $x=U_{d} / U_{0}=\mathcal{V}_{1} / s_{1}$, so that amplification is possible only for $\mathcal{V}_{1}>s_{1}$. At the same time, our experimentally determined plasma velocity $\left(s_{1} \approx 3.2 \times 10^{8} \mathrm{~cm} / \mathrm{s}\right)$ is higher than the Fermi velocity in graphene. The latter represents the upper limit for drift velocity. Therefore, in our experiments, $\mathcal{V}_{1} \ll s_{1}$.

In Ref. [64], instability in a gated plasmonic crystal is predicted for the case when the drift velocity exceeds the plasma wave velocity in the passive region. This mechanism, however, was developed for an ideal system with very low momentum relaxation rate $\gamma_{2} \ll s_{2} / L$ and cannot be applied for our system, where experimental conditions correspond to inequality (13). Also, since experimental data show strong evidence that the threshold value of velocity corresponding to the onset of amplification is smaller than the plasma wave velocity in the active region, we can exclude other mechanisms related to Cherenkov-like plasmon instability [49,59,68-70].

There are only a few theoretical predictions of plasma instability for drift velocities smaller than the plasma velocity. Most known is the instability based on amplified plasmon reflection at the cavity boundaries [8]. This instability is called Dyakonov-Shur instability. The evolution of a dc response with the current increasing and approaching the instability threshold is described in Ref. [67]. It is predicted that the current leads to a decrease of the damping rate in the channel, which turns to zero on the instability threshold [see Eqs. (59) and (60) of Ref. [67]]. Evidently, such a scenario is not realized in our experiment, where the width of resonances does not essentially depend on the dc current.

Although one cannot fully exclude the mechanism based on so-called transit time instability (it is caused by the exchange of excitation between active regions due to 
carriers moving with saturated drift velocity in passive regions) considered in Refs. [71-73], there are some experimental facts against this mechanism. Most importantly, this mechanism implies a strong connection between active regions via the exchange of excitation traveling through passive regions. At the same time, the experimental data show that active regions behave like independent plasmonic resonators.

Importantly, in our work, we do not suggest an instability mechanism. Instead, we predict that current-driven amplification of the incoming radiation is possible without developing any kind of plasma instabilities. Then, limitations $\mathcal{V}_{1}>s_{1}$ (needed for Cherenkov instability) and/or built-in asymmetry of the device (needed for well-known Dyakonov-Shur instability [8]) are lifted. We demonstrate theoretically that amplification is possible even if the drift velocity in the active region is smaller than the plasma wave velocity $\left(\mathcal{V}_{1}<s_{1}\right)$.

Let us now discuss possible reasons why the derived equations do not give the experimentally observed transparency gap. As we demonstrate, in the absence of dissipation, for $s_{1} \gg s_{2}$, and for zero current, dispersion equation (12) reduces to the product of two sines, which give resonance excitation frequencies. One can show that, in the presence of dissipation and for nonzero current, the argument of sine corresponding to the active region transforms as follows:

$$
\omega T_{1} \rightarrow \frac{L_{1} \sqrt{s_{1}^{2} \omega\left(\omega+i \gamma_{1}\right)-\gamma_{1}^{2} \mathcal{V}_{1}^{2} / 4}}{s_{1}^{2}-\mathcal{V}_{1}^{2}} .
$$

To find the fundamental mode frequency, one should equal this expression to $\pi$, which yields $\omega_{1}=\omega_{1}^{\prime}+i \omega_{1}^{\prime \prime}$, where $\omega_{1}^{\prime \prime}=-\gamma / 2$ and $\left(\omega_{1}^{\prime}\right)^{2}=\left(\pi s_{1} / L_{1}\right)^{2}\left(1-x^{2}\right)^{2}-\gamma_{1}^{2}\left(1-x^{2}\right) / 4$. Hence, $\left(\omega_{1}^{\prime}\right)^{2}$ turns to zero for $x=x_{*}$, where

$$
x_{*}=\frac{\mathcal{V}_{1}^{*}}{s_{1}}=\sqrt{1-\left(\frac{\gamma_{1} L_{1}}{2 \pi s_{1}}\right)^{2}}<1 .
$$

When $x$ exceeds $x_{*}$, the solution for $\omega_{1}$ disappears, because $\left(\omega_{1}^{\prime}\right)^{2}$ cannot be negative. Therefore, $x_{*}$ corresponds to the onset of the transparency gap. Actually, this gap does not have a sharp edge, because $\omega_{1}^{\prime \prime} \neq 0$.

The latter property is similar to our experimental observations. Indeed, Fig. (10) shows a gap for the center of resonance peak. In fact, this peak is broadened due to the scattering within the sufficiently large frequency interval about $1 \mathrm{THz}$ (see Fig. 9, where smearing of resonances is shown in different shades of colors). This gap does not show up in our theory, because we are able to perform calculations only for the resonance case $\gamma_{1} \ll \pi s_{1} / L_{1}$. In this case, $x_{*}$ is larger than the critical value of the dissipation-amplification transition, $x_{0}=1 / \sqrt{3}$ (see Fig. 15).
We also note that our calculations are based on the assumption $s_{1} \gg s_{2}$, which is not well fulfilled in our experiment. Actually, in experiment, the mismatch parameter $Z$ entering Eq. (12) is not large (and, as one can show, depends on the current). This result should be taken into account when calculating the band structure of the plasmonic crystal and determining the transparency gap.

A more detailed analysis allowing one to consider the case $s_{1} \sim s_{2}$ and take into account that parameter $\gamma_{1} L_{1} / 2 \pi s_{1}$ is not very small leads to very cumbersome equations that can be analyzed only numerically. It is the subject of ongoing work, and its results will be published elsewhere [74].

Finally, we note that an interesting issue for future study is the response to the radiation with parallel polarization. As we mention above, such radiation does not lead to the redistribution of charge even in the case of nonzero dc current in the perpendicular direction, so that plasmonic effects do not show up. What is nontrivial in this case is the role of the viscosity of the electron liquid in the channel [75-77]. Indeed, because of different velocities in the passive and active strips (due to the difference of scattering times), one can expect viscosity-dependent friction between active and passive regions. This result might lead to arising of Poiseuille-like flows oscillating with the radiation frequency [77].

\section{CONCLUSION}

We have presented a study of gate and drain biasing on resonant 2D Dirac plasmons, excited by $\mathrm{THz}$ radiation, in grating-gate graphene field-effect transistor structures. These grating-gate structures were used to create a periodic structure of highly conducting active regions separated by low-conducting passive regions.

Without applied current, we observed the 2D plasmons with frequencies corresponding to the cavity modes excited under active grating-gate fingers. We have experimentally demonstrated that, at the zero drain bias conditions, active regions act as independent ones. Specifically, we found that excited plasmons follow both the scaling laws of plasmon dispersion and the gate-voltage dependencies predicted by standard physical models developed for a single active cavity.

The experimental results showed strong asymmetry of plasmonic resonances. The shape of such asymmetric resonances was very well fitted by the so-called damped oscillator simplified model, which, in turn, was in very good agreement with the exact calculation of plasmonic dissipation peaks in the active regions.

In the experiments with applied dc current, we observed that an increase of the current leads to a strong Doppler-like shift of the plasma resonances, followed by full transparency and $\mathrm{THz}$ light amplification phenomena.

To interpret the experimental data, we have developed a phenomenological model of a plasmonic crystal formed by 
high and low plasma velocity regions (active and passive regions, respectively). The model captures the basic physics of the problem and allows a qualitative physical explanation of key experimental data. In the case of the zero current, it provided an explanation for why the multiple high carrier density graphene cavities (model active regions) formed under gate fingers respond in the experiments with $\mathrm{THz}$ excitation as independent singlecavity resonators with the resonant frequency defined mainly by their dimensions. The main effect of the passive regions is to provide the plasmon leakage from the active regions, which explains the linewidths of the observed plasmon resonances.

For nonzero current, the phenomenological model describes basic tendencies together with a qualitative description of the physical mechanism of amplification taking place even for drift velocities smaller than the plasma velocities. The origin of the amplification phenomena is proposed as due to the influence of the dc current on the phase shift between the carrier density and drift velocity induced by the $\mathrm{THz}$ radiation.

The presented model captures the main trends and basic physics of the observed phenomena, but it does not provide quantitatively complete descriptions of all observed facts. It shows certain levels of discrepancies on the critical velocity and the threshold frequencies of the dissipation to amplification transition, predicting them much higher than the levels observed in the experiments. Also, the model is not describing the transparency frequency intervals and does not demonstrate the origin of the interpolating Eq. (10) that provides an excellent fit of frequency versus drain voltage experimental dependencies for all cavities. Therefore, our results show a clear need for a further, more advanced theory of plasmonic crystals.

In summary, we list our main results.

(i) We have demonstrated current-driven excitation of Dirac plasmons in grating-gate graphene-channel transistor nanostructures leading to $\mathrm{THz}$ radiation amplification up to room-temperature. Specifically, we demonstrated that the plasmon resonances are redshifted and undergo complete transparency to electromagnetic radiation followed by the amplification with more than $9 \%$ of gain and blueshift of the resonant plasmon frequency in the $\mathrm{THz}$ range.

(ii) We have theoretically modeled the 2D Dirac plasmon system to explain the origin of the amplification phenomena as due to the influence of dc current on the phase shift between the change of carrier density and drift velocity induced by the $\mathrm{THz}$ radiation. This radiation-induced correction to dissipation is sensitive to this phase shift, and with an increasing dc current the dissipation becomes negative, which captures the basic physics behind the experimentally observed switching from dissipation to amplification. (iii) We have demonstrated, both theoretically and experimentally, that the transition to the amplification regime occurs at drift velocities smaller than the plasma wave velocity. We theoretically found a critical value of drift velocity corresponding to the onset of amplification.

(iv) We have discussed a leakage of plasma waves in the plasmonic crystals, which can limit the plasmonic quality factor even in very clean structures. We derived an analytical expression for leakagedominated damping which shows that leakage can be controlled by gates covering passive regions of the plasmonic crystal.

Finally, we stress that all experimental results presented in this work were obtained at roomtemperature. We obtained amplification of the order of $10 \%$ that is far beyond the level of the fundamental limit for interband-transition-originated gain in monolayer graphene. Therefore, despite lacking a complete quantitative theoretical description, this work demonstrating room-temperature $\mathrm{THz}$ amplification paves the way toward a $\mathrm{THz}$ plasmonic technology with a new generation of allelectronic, resonant, voltage- and current-tunable $\mathrm{THz}$ amplifiers.

\section{ACKNOWLEDGMENTS}

We thank V. Ryzhii, S. Mikhailov, D. Svintsov, K. Maussang, M. S. Shur, and M. Dyakonov for many useful discussions. The work was supported by JSPS KAKENHI (No. 16H06361, No. 16K14243, and No. 18H05331), Japan; the International Research Agendas program of the Foundation for Polish Science cofinanced by the European Union under the European Regional Development Fund for CENTERA (No. MAB/2018/9); and the Foundation for Polish Science through the TEAM Project No. POIR.04.04.00-00-3D76/16 (TEAM/ 2016-3/25). The work of V. K. was supported by Russian Foundation of Basic Research (Grant No. 20-02-00490) and by Foundation for the Advancement of Theoretical Physics and Mathematics BASIS. The work of V. V. P. was carried out within the framework of the state task.

[1] A. V. Chaplik, Possible Crystallization of Charge Carriers in Low-Density Inversion Layers, Zh. Eksp. Teor. Fiz. 62, 746 (1972) [, Sov. Phys. JETP 35, 395 (1972)].

[2] S. J. Allen, D. C. Tsui, and R. A. Logan, Observation of the Two-Dimensional Plasmon in Silicon Inversion Layers, Phys. Rev. Lett. 38, 980 (1977).

[3] T. Theis, J. Kotthaus, and P. Stiles, Two-Dimensional Magnetoplasmon in the Silicon Inversion Layer, Solid State Commun. 24, 273 (1977).

[4] D. Tsui, S. Allen, R. Logan, A. Kamgar, and S. Coppersmith, High Frequency Conductivity in Silicon Inversion Layers: 
Drude Relaxation, 2D Plasmons and Minigaps in a Surface Superlattice, Surf. Sci. 73, 419 (1978).

[5] T. Theis, J. Kotthaus, and P. Stiles, Wavevector Dependence of the Two-Dimensional Plasmon Dispersion Relationship in the (100) Silicon Inversion Layer, Solid State Commun. 26, 603 (1978).

[6] T. N. Theis, Plasmons in Inversion Layers, Surf. Sci. 98, 515 (1980).

[7] D. Tsui, E. Gornik, and R. Logan, Far Infrared Emission from Plasma Oscillations of Si Inversion Layers, Solid State Commun. 35, 875 (1980).

[8] M. Dyakonov and M. Shur, Shallow Water Analogy for a Ballistic Field Effect Transistor: New Mechanism of Plasma Wave Generation by dc Current, Phys. Rev. Lett. 71, 2465 (1993).

[9] M. Dyakonov and M. Shur, Detection, Mixing, and Frequency Multiplication of Terahertz Radiation by Two-Dimensional Electronic Fluid, IEEE Trans. Electron Devices 43, 380 (1996).

[10] K. Hirakawa, K. Yamanaka, M. Grayson, and D. C. Tsui, Far-Infrared Emission Spectroscopy of Hot TwoDimensional Plasmons in $\mathrm{Al}_{0.3} \mathrm{Ga}_{0.7} \mathrm{As} / \mathrm{GaAs}$ Heterojunctions, Appl. Phys. Lett. 67, 2326 (1995).

[11] W. Knap, J. Lusakowski, T. Parenty, S. Bollaert, A. Cappy, V. V. Popov, and M. S. Shur, Terahertz Emission by Plasma Waves in $60 \mathrm{~nm}$ Gate High Electron Mobility Transistors, Appl. Phys. Lett. 84, 2331 (2004).

[12] J. Lusakowski, W. Knap, N. Dyakonova, L. Varani, J. Mateos, T. Gonzalez, Y. Roelens, S. Bollaert, A. Cappy, and K. Karpierz, Voltage Tuneable Terahertz Emission from a Ballistic Nanometer InGaAs/InAlAs Transistor, J. Appl. Phys. 97, 064307 (2005).

[13] N. Dyakonova, F. Teppe, J. Łusakowski, W. Knap, M. Levinshtein, A. P. Dmitriev, M. S. Shur, S. Bollaert, and A. Cappy, Magnetic Field Effect on the Terahertz Emission from Nanometer InGaAs/AlInAs High Electron Mobility Transistors, J. Appl. Phys. 97, 114313 (2005).

[14] T. Otsuji, Y. M. Meziani, T. Nishimura, T. Suemitsu, W. Knap, E. Sano, T. Asano, and V. V. Popov, Emission of Terahertz Radiation from Dual Grating Gate PlasmonResonant Emitters Fabricated with InGaP/InGaAs/GaAs Material Systems, J. Phys. Condens. Matter 20, 384206 (2008).

[15] S. Boubanga-Tombet, F. Teppe, J. Torres, A. El Moutaouakil, D. Coquillat, N. Dyakonova, C. Consejo, P. Arcade, P. Nouvel, H. Marinchio, T. Laurent, C. Palermo, A. Penarier, T. Otsuji, L. Varani, and W. Knap, Room Temperature Coherent and Voltage Tunable Terahertz Emission from Nanometer-Sized Field Effect Transistors, Appl. Phys. Lett. 97, 262108 (2010).

[16] A. El Fatimy, N. Dyakonova, Y. Meziani, T. Otsuji, W. Knap, S. Vandenbrouk, K. Madjour, D. Théron, C. Gaquiere, M. A. Poisson, S. Delage, P. Prystawko, and C. Skierbiszewski, AlGaN/GaN High Electron Mobility Transistors as a Voltage-Tunable Room Temperature Terahertz. Sources, J. Appl. Phys. 107, 024504 (2010).

[17] W. Knap, S. Nadar, H. Videlier, S. Boubanga-Tombet, D. Coquillat, N. Dyakonova, F. Teppe, K. Karpierz, J. Łusakowski, M. Sakowicz, I. Kasalynas, D. Seliuta, G. Valusis, T. Otsuji, Y. Meziani, A. El Fatimy, S. Vandenbrouk,
K. Madjour, D. Théron, and C. Gaquière, Field Effect Transistors for Terahertz Detection and Emission, J. Infrared Millimeter Terahertz Waves 32, 618 (2011).

[18] T. Otsuji, T. Watanabe, S. A. B. Tombet, A. Satou, W. M. Knap, V. V. Popov, M. Ryzhii, and V. Ryzhii, Emission and Detection of Terahertz Radiation Using Two-Dimensional Electrons in IIIV Semiconductors and Graphene, IEEE Trans. Terahertz Sci. Technol. 3, 63 (2013).

[19] A. V. Muravjov, D. B. Veksler, V. V. Popov, O. V. Polischuk, N. Pala, X. Hu, R. Gaska, H. Saxena, R. E. Peale, and M. S. Shur, Temperature Dependence of Plasmonic Terahertz Absorption in Grating-Gate Gallium-Nitride Transistor Structures, Appl. Phys. Lett. 96, 042105 (2010).

[20] Y. Kurita, G. Ducournau, D. Coquillat, A. Satou, K. Kobayashi, S. B. Tombet, Y. M. Meziani, V. V. Popov, W. Knap, T. Suemitsu, and T. Otsuji, Ultrahigh Sensitive Sub-terahertz Detection by INP-Based Asymmetric DualGrating-Gate High-Electron-Mobility Transistors and Their Broadband Characteristics, Appl. Phys. Lett. 104, 251114 (2014).

[21] S. Boubanga-Tombet, Y. Tanimoto, A. Satou, T. Suemitsu, Y. Wang, H. Minamide, H. Ito, D. V. Fateev, V. V. Popov, and T. Otsuji, Current-Driven Detection of Terahertz. Radiation Using a Dual-Grating-Gate Plasmonic Detector, Appl. Phys. Lett. 104, 262104 (2014).

[22] P. Faltermeier, P. Olbrich, W. Probst, L. Schell, T. Watanabe, S. A. Boubanga-Tombet, T. Otsuji, and S. D. Ganichev, Helicity Sensitive Terahertz Radiation Detection by DualGrating-Gate High Electron Mobility Transistors, J. Appl. Phys. 118, 084301 (2015).

[23] V. A. Shalygin, M. D. Moldavskaya, M. Y. Vinnichenko, K. V. Maremyanin, A. A. Artemyev, V. Y. Panevin, L. E. Vorobjev, D. A. Firsov, V. V. Korotyeyev, A. V. Sakharov, E. E. Zavarin, D. S. Arteev, W. V. Lundin, A. F. Tsatsulnikov, S. Suihkonen, and C. Kauppinen, Selective Terahertz Emission due to Electrically Excited 2D Plasmons in AlGaN/GaN Heterostructure, J. Appl. Phys. 126, 183104 (2019).

[24] A. H. C. Neto, F. Guinea, N. M. R. Peres, K. S. Novoselov, and A. K. Geim, The Electronic Properties of Graphene, Rev. Mod. Phys. 81, 109 (2009).

[25] F. Bonaccorso, Z. Sun, T. Hasan, and A. C. Ferrari, Graphene Photonics and Optoelectronics, Nat. Photonics 4, 611 (2010).

[26] O. Vafek, Thermoplasma Polariton within Scaling Theory of Single-Layer Graphene, Phys. Rev. Lett. 97, 266406 (2006).

[27] V. Ryzhii, Terahertz Plasma Waves in Gated Graphene Heterostructures, Jpn. J. Appl. Phys. 45, L923 (2006).

[28] E. H. Hwang and S. D. Sarma, Dielectric Function, Screening, and Plasmons in Two-Dimensional Graphene, Phys. Rev. B 75, 205418 (2007).

[29] V. Ryzhii, A. Satou, and T. Otsuji, Plasma Waves in TwoDimensional Electron-Hole System in Gated Graphene Heterostructures, J. Appl. Phys. 101, 024509 (2007).

[30] M. Jablan, H. Buljan, and M. Soljačić, Plasmonics in Graphene at Infrared Frequencies, Phys. Rev. B 80, 245435 (2009).

[31] P. West, S. Ishii, G. Naik, N. Emani, V. Shalaev, and A. Boltasseva, Searching for Better Plasmonic Materials, Laser Photonics Rev. 4, 795 (2010). 
[32] A. N. Grigorenko, M. Polini, and K. S. Novoselov, Graphene Plasmonics, Nat. Photonics 6, 749 (2012).

[33] F. H. L. Koppens, D. E. Chang, and F. J. G. de Abajo, Graphene Plasmonics: A Platform for Strong Light-Matter Interactions, Nano Lett. 11, 3370 (2011).

[34] Y.-M. Bahk, G. Ramakrishnan, J. Choi, H. Song, G. Choi, Y. H. Kim, K. J. Ahn, D.-S. Kim, and P. C. M. Planken, Plasmon Enhanced Terahertz Emission from Single Layer Graphene, ACS Nano 8, 9089 (2014).

[35] R. DeglInnocenti, D. S. Jessop, C. W. O. Sol, L. Xiao, S. J. Kindness, H. Lin, J. A. Zeitler, P. Braeuninger-Weimer, S. Hofmann, Y. Ren, V. S. Kamboj, J. P. Griffiths, H. E. Beere, and D. A. Ritchie, Fast Modulation of Terahertz Quantum Cascade Lasers Using Graphene Loaded Plasmonic Antennas, ACS Photonics 3, 464 (2016).

[36] S. Chakraborty, O. P. Marshall, T. G. Folland, Y.-J. Kim, A. N. Grigorenko, and K. S. Novoselov, Gain Modulation by Graphene Plasmons in Aperiodic Lattice Lasers, Science 351, 246 (2016).

[37] X. Cai, A. B. Sushkov, M. M. Jadidi, L. O. Nyakiti, R. L. Myers-Ward, D. K. Gaskill, T. E. Murphy, M. S. Fuhrer, and H. D. Drew, Plasmon-Enhanced Terahertz Photodetection in Graphene, Nano Lett. 15, 4295 (2015).

[38] A. Bylinkin, E. Titova, V. Mikheev, E. Zhukova, S. Zhukov, M. Belyanchikov, M. Kashchenko, A. Miakonkikh, and D. Svintsov, Tight-Binding Terahertz Plasmons in ChemicalVapor-Deposited Graphene, Phys. Rev. Applied 11, 054017 (2019).

[39] D. Svintsov, Emission of Plasmons by Drifting Dirac Electrons: A Hallmark of Hydrodynamic Transport, Phys. Rev. B 100, 195428 (2019).

[40] P. Olbrich, J. Kamann, M. König, J. Munzert, L. Tutsch, J. Eroms, D. Weiss, M.-H. Liu, L. E. Golub, E. L. Ivchenko, V. V. Popov, D. V. Fateev, K. V. Mashinsky, F. Fromm, T. Seyller, and S. D. Ganichev, Terahertz Ratchet Effects in Graphene with a Lateral Superlattice, Phys. Rev. B 93, 075422 (2016).

[41] D. V. Fateev, K. V. Mashinsky, O. V. Polischuk, and V. V. Popov, Excitation of Propagating Plasmons in a Periodic Graphene Structure by Incident Terahertz Waves, Phys. Rev. Applied 11, 064002 (2019).

[42] T. Watanabe, T. Fukushima, Y. Yabe, S. A. B. Tombet, A. Satou, A. A. Dubinov, V. Y. Aleshkin, V. Mitin, V. Ryzhii, and T. Otsuji, The Gain Enhancement Effect of Surface Plasmon Polaritons on Terahertz Stimulated Emission in Optically Pumped Monolayer Graphene, New J. Phys. 15, 075003 (2013).

[43] Y. Takatsuka, K. Takahagi, E. Sano, V. Ryzhii, and T. Otsuji, Gain Enhancement in Graphene Terahertz Amplifiers with Resonant Structures, J. Appl. Phys. 112, 033103 (2012).

[44] S. Boubanga-Tombet, S. Chan, T. Watanabe, A. Satou, V. Ryzhii, and T. Otsuji, Ultrafast Carrier Dynamics and Terahertz Emission in Optically Pumped Graphene at Room Temperature, Phys. Rev. B 85, 035443 (2012).

[45] V. V. Popov, D. V. Fateev, T. Otsuji, Y. M. Meziani, D. Coquillat, and W. Knap, Plasmonic Terahertz Detection by a Double-Grating-Gate Field-Effect Transistor Structure with an Asymmetric Unit Cell, Appl. Phys. Lett. 99, 243504 (2011).
[46] R. J. Wilkinson, C. D. Ager, T. Duffield, H. P. Hughes, D. G. Hasko, H. Ahmed, J. E. F. Frost, D. C. Peacock, D. A. Ritchie, G. A. C. Jones, C. R. Whitehouse, and N. Apsley, Plasmon Excitation and Self-Coupling in a Biperiodically Modulated Two-Dimensional Electron Gas, J. Appl. Phys. 71, 6049 (1992).

[47] D. Coquillat, S. Nadar, F. Teppe, N. Dyakonova, S. Boubanga-Tombet, W. Knap, T. Nishimura, T. Otsuji, Y. M. Meziani, G. M. Tsymbalov, and V. V. Popov, Room Temperature Detection of Sub-terahertz Radiation in Double-Grating-Gate Transistors, Opt. Express 18, 6024 (2010).

[48] A. Satou, G. Tamamushi, K. Sugawara, J. Mitsushio, V. Ryzhii, and T. Otsuji, A Fitting Model for Asymmetric I-V Characteristics of Graphene FETs for Extraction of Intrinsic Mo, IEEE Trans. Electron Devices 63, 3300 (2016).

[49] S. A. Mikhailov, Plasma Instability and Amplification of Electromagnetic Waves in Low-Dimensional Electron Systems, Phys. Rev. B 58, 1517 (1998).

[50] L. Ju, B. Geng, J. Horng, C. Girit, M. Martin, Z. Hao, H. A. Bechtel, X. Liang, A. Zettl, Y. R. Shen, and F. Wang, Graphene Plasmonics for Tunable Terahertz Metamaterials, Nat. Nanotechnol. 6, 630 (2011).

[51] See Supplemental Material at http://link.aps.org/ supplemental/10.1103/PhysRevX.10.031004 for calculating plasmon-assisted dissipation in an array of highly conducting strips separated by low-conducting regions and discuss corresponding correction to the radiation transmission coefficient through this structure.

[52] H. Yan, X. Li, B. Chandra, G. Tulevski, Y. Wu, M. Freitag, W. Zhu, P. Avouris, and F. Xia, Tunable Infrared Plasmonic Devices Using Graphene/Insulator Stacks, Nat. Nanotechnol. 7, 330 (2012).

[53] H. Yan, Z. Li, X. Li, W. Zhu, P. Avouris, and F. Xia, Infrared Spectroscopy of Tunable Dirac Terahertz MagnetoPlasmons in Graphene, Nano Lett. 12, 3766 (2012).

[54] H. Yan, T. Low, W. Zhu, Y. Wu, M. Freitag, X. Li, F. Guinea, P. Avouris, and F. Xia, Damping Pathways of Mid-infrared Plasmons in Graphene Nanostructures, Nat. Photonics 7, 394 (2013).

[55] A. Laturia, M. L. Van de Put, and W. G. Vandenberghe, Dielectric Properties of Hexagonal Boron Nitride and Transition Metal Dichalcogenides: From Monolayer to Bulk, npj 2D Mater. Appl. 2, 6 (2018).

[56] R. Geick, C. H. Perry, and G. Rupprecht, Normal Modes in Hexagonal Boron Nitride, Phys. Rev. 146, 543 (1966).

[57] C. R. Dean, A. F. Young, I. Meric, C. Lee, L. Wang, S. Sorgenfrei, K. Watanabe, T. Taniguchi, P. Kim, K. L. Shepard, and J. Hone, Boron Nitride Substrates for HighQuality Graphene Electronics, Nat. Nanotechnol. 5, 722 (2010).

[58] G. L. Yu, R. Jalil, B. Belle, A. S. Mayorov, P. Blake, F. Schedin, S. V. Morozov, L. A. Ponomarenko, F. Chiappini, S. Wiedmann, U. Zeitler, M. I. Katsnelson, A. K. Geim, K. S. Novoselov, and D. C. Elias, Interaction Phenomena in Graphene Seen through Quantum Capacitance, Proc. Natl. Acad. Sci. U.S.A. 110, 3282 (2013).

[59] C. Hwang, D. A. Siegel, S.-K. Mo, W. Regan, A. Ismach, Y. Zhang, A. Zettl, and A. Lanzara, Fermi Velocity Engineer- 
ing in Graphene by Substrate Modification, Sci. Rep. 2, 590 (2012).

[60] A. Tomasino, A. Parisi, S. Stivala, P. Livreri, A. C. Cino, A. C. Busacca, M. Peccianti, and R. Morandotti, Wideband $\mathrm{THz}$ Time Domain Spectroscopy Based on Optical Rectification and Electro-Optic Sampling, Sci. Rep. 3, 3116 (2013).

[61] J. H. Strait, P. Nene, W.-M. Chan, C. Manolatou, S. Tiwari, F. Rana, J. W. Kevek, and P. L. McEuen, Confined Plasmons in Graphene Microstructures: Experiments and Theory, Phys. Rev. B 87, 241410(R) (2013).

[62] H. Yan, F. Xia, Z. Li, and P. Avouris, Plasmonics of Coupled Graphene Micro-structures, New J. Phys. 14, 125001 (2012).

[63] K. M. Daniels, M. M. Jadidi, A. B. Sushkov, A. Nath, A. K. Boyd, K. Sridhara, H. D. Drew, T. E. Murphy, R. L. MyersWard, and D. K. Gaskill, Narrow Plasmon Resonances Enabled by Quasi-Freestanding Bilayer Epitaxial Graphene, 2D Mater. 4, 025034 (2017).

[64] V. Y. Kachorovskii and M. S. Shur, Current-Induced Terahertz Oscillations in Plasmonic Crystal, Appl. Phys. Lett. 100, 232108 (2012).

[65] V. V. Popov, O. V. Polischuk, W. Knap, and A. El Fatimy, Broadening of the Plasmon Resonance due to Plasmon-Plasmon Intermode Scattering in Terahertz High-Electron-Mobility Transistors, Appl. Phys. Lett. 93, 263503 (2008).

[66] I. V. Rozhansky, V. Y. Kachorovskii, and M. S. Shur, Helicity-Driven Ratchet Effect Enhanced by Plasmons, Phys. Rev. Lett. 114, 246601 (2015).

[67] D. Veksler, F. Teppe, A. P. Dmitriev, V. Y. Kachorovskii, W. Knap, and M. S. Shur, Detection of Terahertz Radiation in Gated Two-Dimensional Structures Governed by dc Current, Phys. Rev. B 73, 125328 (2006).

[68] O. Matov, O. Meshkov, O. Polischuk, and V. Popov, Generation of Submillimeter Electromagnetic Radiation from Two-Dimensional Plasma Waves in a Semiconductor
Heterostructure with Metal Grating, Physica (Amsterdam) 241A, 409 (1997).

[69] M. V. Krasheninnikov and A. V. Chaplik, Instabilities of Two-Dimensional Plasma Waves, Zh. Eksp. Teor. Fiz 79, 555 (1980) [Sov. Phys. JETP 52, 279 (1980)].

[70] G. R. Aizin, J. Mikalopas, and M. Shur, Current-Driven Plasmonic Boom Instability in Three-Dimensional Gated Periodic Ballistic Nanostructures, Phys. Rev. B 93, 195315 (2016).

[71] V. Ryzhii, A. Satou, and M. S. Shur, Transit Time Mechanism of Plasma Instability in High Electron Mobility Transistors, Phys. Status Solidi A 202, R113 (2005).

[72] V. Ryzhii, A. Satou, and M. S. Shur, Plasma Instability and Terahertz Generation in HEMTs due to Electron TransitTime Effect, Trans. Inst. Electron., Inf. Commun. Eng., Sect. E 89, 1012 (2006).

[73] Y. Koseki, V. Ryzhii, T. Otsuji, V. V. Popov, and A. Satou, Giant Plasmon Instability in a Dual-Grating-Gate Graphene Field-Effect Transistor, Phys. Rev. B 93, 245408 (2016).

[74] I. V. Gorbenko, V. Y. Kachorovskii, and W. Knap (private communication).

[75] I. Torre, A. Tomadin, A. K. Geim, and M. Polini, Nonlocal Transport and the Hydrodynamic Shear Viscosity in Graphene, Phys. Rev. B 92, 165433 (2015).

[76] D. A. Bandurin, I. Torre, R. K. Kumar, M. Ben Shalom, A. Tomadin, A. Principi, G. H. Auton, E. Khestanova, K. S. Novoselov, I. V. Grigorieva, L. A. Ponomarenko, A. K. Geim, and M. Polini, Negative Local Resistance Caused by Viscous Electron Backflow in Graphene, Science 351, 1055 (2016).

[77] J. A. Sulpizio, L. Ella, A. Rozen, J. Birkbeck, D. J. Perello, D. Dutta, M. Ben-Shalom, T. Taniguchi, K. Watanabe, T. Holder, R. Queiroz, A. Principi, A. Stern, T. Scaffidi, A. K. Geim, and S. Ilani, Visualizing Poiseuille Flow of Hydrodynamic Electrons, Nature (London) 576, 75 (2019). 